\title{
Combined analysis of Planck and SPTPol data favors the early dark energy models
}

\author{
Anton Chudaykin, ${ }^{1 a, b}$ Dmitry Gorbunov, ${ }^{2 a, b}$ Nikita Nedelko ${ }^{3 a, c}$ \\ ${ }^{a}$ Institute for Nuclear Research of the Russian Academy of Sciences, \\ 60th October Anniversary Prospect, 7a, 117312 Moscow, Russia \\ ${ }^{b}$ Moscow Institute of Physics and Technology, \\ Institutsky lane 9, Dolgoprudny, Moscow region, 141700, Russia \\ ${ }^{c}$ Department of Particle Physics and Cosmology, Physics Faculty, \\ M.V. Lomonosov Moscow State University, \\ Vorobjevy Gory, 119991 Moscow, Russia
}

\begin{abstract}
We study the implications of the Planck temperature power spectrum at low multipoles, $\ell<1000$, and SPTPol data. We show that this combination predicts consistent lensing-induced smoothing of acoustic peaks within $\Lambda$ CDM cosmology and yields the robust predictions of the cosmological parameters. Combining only the Planck large-scale temperature data and the SPTPol polarization and lensing measurements within $\Lambda$ CDM model we found substantially lower values of linear matter density perturbation $\sigma_{8}$ which bring the late-time parameter $S_{8}=\sigma_{8} \sqrt{\Omega_{m} / 0.3}=$ $0.763 \pm 0.022$ into accordance with galaxy clustering and weak lensing measurements. It also raises up the Hubble constant $H_{0}=69.68 \pm 1.00 \mathrm{~km} \mathrm{~s}^{-1} \mathrm{Mpc}^{-1}$ that reduces the Hubble tension to the $2.5 \sigma$ level. We examine the residual tension in the Early Dark Energy (EDE) model which produces the brief energy injection prior to recombination. We implement both the background and perturbation evolutions of the scalar field which potential scales as $V(\phi) \propto \phi^{2 n}$. Including cosmic shear measurements (KiDS, VIKING-450, DES) and local distance-ladder data (SH0ES) to the combined fit we found that EDE completely alleviates the Hubble tension while not degradating the fit to large-scale structure data. The EDE scenario significantly improves the goodness-of-fit by $2.9 \sigma$ in comparison with the concordance $\Lambda$ CDM model. The account for the intermediate-redshift data (the supernova dataset and baryon acoustic oscillation data) fits perfectly to our parameter predictions and indicates the preference of EDE over $\Lambda$ CDM at $3 \sigma$.
\end{abstract}

\footnotetext{
${ }^{1}$ chudy@ms 2 .inr.ac.ru

${ }^{2}$ gorby@ms 2. inr . ac.ru

${ }^{3}$ nikita.nedelko1999@gmail.com
} 


\section{Contents}

1 Introduction 1

2 Data sets and numerical procedure $\quad 7$

2.1 Cosmological data $\quad 7$

2.2 Numerical procedure 8

3 Methodology and $\Lambda$ CDM constraints 9

$\begin{array}{lll}3.1 & A_{L} \text { test } & 12\end{array}$

4 Early Dark Energy model 13

$\begin{array}{ll}4.1 \text { Background dynamics } & 13\end{array}$

$\begin{array}{lll}4.2 & \text { Perturbed dynamics } & 15\end{array}$

5 Cosmological constraints within the Early Dark Energy model 17

$\begin{array}{ll}5.1 \text { Parameter constraints for } n=3 & 18\end{array}$

5.2 Promoting $n$ to a free parameter 24

6 Conclusion 26

\section{Introduction}

Measurements of the cosmic microwave background (CMB) have provided a profound insight into the nature of the Universe. Temperature and polarization anisotropies of the CMB encode detailed information about the composition and evolution of our Universe. Indeed, the primary $\mathrm{CMB}$ is one of the most powerful probes of the early-Universe cosmology coming from the last scattering. It also provides cosmological (model-dependent) probe of the late-Universe composition through observation of the angular scale of the sound horizon at last scattering $\theta_{s}$. Besides, the latetime physics leaves distinctive imprints on the CMB anisotropy at small angular scales. In particular, the small scale distortions caused by gravitational lensing [1] and Sunyaev-Zeldovich effect [2] provide a unique probe of the Universe evolution at late times. Our capability in tackling all these subtle effects strongly depends on the quality and self-consistency of observed CMB maps.

The most profound observation of CMB anisotropy over the full sky has been accomplished by the Planck satellite [3]. This mission provides with measurements of cosmological parameters at the percent level accuracy thus exemplifying the potential of CMB surveys as a high-precision probe of cosmology. However, the extensive 
analyses of the plethora of CMB data from this spacecraft have revealed internal tensions within the Planck dataset. The most prominent feature often called 'lensing tension' refers to overly enhanced lensing smoothing of the CMB peaks compared to the $\Lambda$ CDM expectation [3-6]. A recently developed technique for probing the gravitational lensing potential from CMB data in a model-independent way reveals $2.8 \sigma$ tension between the full Planck dataset and $\Lambda$ CDM expectation [7]. This anomaly raises the issue of whether the Planck dataset is internally consistent. The Planck collaboration has thoroughly examined this problem and presented the cosmological parameter constraints from different multipole ranges $[3,6]$. They found a moderate disagreement $(\gtrsim 2 \sigma)$ in parameters extracted from multipoles $\ell<800$ and $\ell>800$ of CMB temperature anisotropy power spectrum (TT). The similar trend in driving cosmological parameters has been revealed for the full Planck dataset including polarization measurements albeit with a lower statistical significance $(\lesssim 2 \sigma)$. Although the Planck collaboration argued that the exclusion of power spectrum at high multipoles does not change their baseline result, its impact might be crucial for extensions of $\Lambda \mathrm{CDM}$ model. A considerably less tension originates from the overall deficit of the Planck TT power spectra across the multipole range $20 \lesssim \ell \lesssim 30$. Remarkably, this feature is strongly disfavoured by extra smoothing of acoustic CMB peaks observed by Planck on small angular scales which pull the amplitude of power spectra to larger values. These internal tensions within the Planck dataset emphasize the need for independent measurement of the CMB anisotropies, especially on small scales.

An important consistency check of CMB anisotropy measurements at small scales can be provided by ground-based telescopes. The small-scale CMB anisotropies can be measured by the South Pole Telescope (SPT) [8-10] and the Atacama Cosmology Telescope (ACT) [11, 12]. The small-scale EE power spectrum has been recently measured by POLARBEAR instrument [13]. These observations supplement the satellite-based measurements since the ground-based telescopes are sensitive to much smaller angular scales unattainable in full sky surveys. The most sensitive measurements to date of small-angular scale temperature anisotropy have been performed by the SPT observation of $2540 \mathrm{deg}^{2}$ SPT-SZ survey [14]. The SPT measurements of CMB temperature anisotropy at multipole range $650<\ell<3000$ augmented with WMAP-7 observations of large angular scales $\ell \lesssim 500$ prefer a slightly smaller lensing amplitude which is at the lower end, but within the $1 \sigma$ prediction of $\Lambda$ CDM model [14]. Thereby, SPT-SZ survey provides consistent temperature power spectrum down to arcminute scales which is compatible with theoretical predictions of the $\Lambda \mathrm{CDM}$ model.

Measurements of $\mathrm{CMB}$ polarization anisotropies is a promising tool to obtain more robust parameter inference. Since E-mode measurements are expected to be fractionally less contaminated by foregrounds than temperature measurements $[15,16]$, the E-mode auto-power spectrum (EE) and the temperature-E-mode correlation (TE) have higher potential to study very small angular scales. In particu- 
lar, polarization measurements demonstrates high sensitivity to the photon diffusion damping tail of the CMB power spectrum enabling tighter constraints on cosmological parameters. Besides, TE and EE power spectra provide a powerful consistency check of lensing smoothing effect which can shed light on the internal tensions in the Planck dataset.

The most accurate measurements to date of TE and EE spectra have been provided by SPTPol analysis of $500 \mathrm{deg}^{2}$ survey [17]. These data are the most sensitive measurements of TE and EE spectra at $\ell>1050$ and $\ell>1475$, respectively. These observations reveal less smoothing of CMB acoustic peaks as allowed by $\Lambda$ CDM which level is $1.4 \sigma$ below the $\Lambda$ CDM expectation and $2.9 \sigma$ lower than the value prefered by the Planck collaboration. It disproves stronger smoothing of the acoustic scales observed in the Planck maps. Altogether, the most sophisticated ground-based experiments provide consistent measurements of the temperature and polarization CMB anisotropies which do not find compelling evidence for the enhanced smoothing of the acoustic peaks. Conversely, they found a mild deficit of the lensing power in the CMB anisotropies on small scales which may be hints of new physics.

Besides measurements of the CMB power spectrum, the lensing potential can be directly extracted from quadratic estimators of $T-, E$ - or $B$-fields. Independent measurement of lensing power spectrum $C_{\ell}^{\phi \phi}$ represents an important cross-check of the CMB anisotropies on small scales and can verify the lensing tension observed in the Planck maps. Lensing potential power spectrum $C_{\ell}^{\phi \phi}$ in the multipole range $100<\ell<2000$ was recently constrained from the 500deg SPTPol survey [18, 19]. This measurement based on a minimum-variance estimator that combines both temperature and polarization CMB maps predicts a $1.8 \sigma$ lower value of lensing power as compared to the Planck $\Lambda$ CDM cosmology. Thereby, SPTPol measurements exhibit similar trends in both CMB power spectra and lensing potential power spectrum probes. Planck collaboration also provides independent measurements of lensing potential power spectrum which is consistent within $1 \sigma$ to the $\Lambda$ CDM expectations [3]. Although Planck lensing likelihood has somewhat higher constraining power [19], we exploit SPTPol lensing measurements in our analysis to extract lensing information entirely from SPTPol survey. It allows us to investigate the deficit of lensing power observed in both TE, EE power spectra and lensing power spectrum $C_{\ell}^{\phi \phi}$ within SPTPol survey in a more consistent way.

Alongside with the CMB measurements which bring only model-dependent parameter constraints in the late Universe, the local measurements support direct extraction of cosmological parameters at low redshifts. These probes also provide another important consistency check of CMB results in a concrete cosmological model. Intriguingly, there is a modest level of discrepancy between the Planck results and direct probes within the $\Lambda \mathrm{CDM}$ model. In particular, the amplitude of linear density fluctuations $\sigma_{8}$ deduced from galaxy cluster counts [20-24], cosmic shear and/or galaxy clustering measurements [25-29] is distinctly lower than the value of $\sigma_{8}$ sug- 
gested by Planck. Combining different cosmic shear surveys (KiDS, VIKING-450 and DES) [30] ${ }^{1}$ leads to the substantially tighter constraint $S_{8}=0.762_{-0.024}^{+0.025}$ where $S_{8}=\sigma_{8} \sqrt{\Omega_{m} / 0.3}$ is the principal-component parameter for weak gravitational lensing analyses. This measurement is in tension with the Planck value $S_{8}=0.830 \pm 0.013$ [3] at the level of $2.5 \sigma$. Recently, this joint analysis has been improved by including additional small-scale information yielding $S_{8}=0.755_{-0.021}^{+0.019}$, that exacerbates the tension with the Planck to $3.2 \sigma$ [31]. In our work, we stick to the joint cosmic shear analysis [30] which applies a more conservative approach of excluding the small-scale information.

More controversial tension between low-redshift and high-redshift data is attributed to the determination of the present-day expansion rate of the Universe. Planck measurements based on CMB temperature, polarization and lensing power spectra accommodate the model-dependent estimate $H_{0}=67.36 \pm 0.54$ [3]. Traditional distance-ladder measurements made up Cepheids and Type Ia Supernovae favour generally higher values of the Hubble constant [32-34]. Upon improved calibration of the Cepheid distance-ladder in the Large Magellanic Cloud, the SH0ES collaboration presents the most severe constraint $H_{0}=74.03 \pm 1.42 \mathrm{~km} \mathrm{~s}^{-1} \mathrm{Mpc}^{-1}$ which tightens the tension with the Planck measurement to $4.4 \sigma$ [35]. Remarkably, the direct measurement based on Type Ia Supernovae is quite robust against the choice of distance indicators. A number of other techniques which has been used to calibrate the SN luminosity distances (distant megamasers [36], Miras or variable red giant stars [37], strongly lensed quasars [38]) gives coherent results competitive with the Cepheid-based measurement [35] albeit with lager $H_{0}$ uncertainties, and only the Tip of the Red Giant Branch (TRGB) as a distance measure brings moderately smaller value of the Hubble constant $H_{0}=69.6 \pm 1.9 \mathrm{~km} \mathrm{~s}^{-1} \mathrm{Mpc}^{-1}$ [39]. Intriguingly, all distance-ladder measurements ${ }^{2}$ are perfectly consistent with a completely different technique of the Hubble constant measurement based on strong gravitational lensing time delays which dictates $H_{0}=73.3_{-1.8}^{+1.7} \mathrm{~km} \mathrm{~s}^{-1} \mathrm{Mpc}^{-1}$ [40]. A cosmology model-independent analysis for a flat Universe accumulating strong gravitation lensing and supernova data supports this estimate yielding $H_{0}=72.8_{-1.7}^{+1.6} \mathrm{~km} \mathrm{~s}^{-1} \mathrm{Mpc}^{-1}$ [41].

Besides direct measurements based on local distance anchors, there is a powerful inverse-distance-ladder approach which makes use of the baryon acoustic oscillations (BAO) measurements and an independent determination of the sound horizon $r_{\text {drag }}$. For the base- $\Lambda \mathrm{CDM}$ model, this approach can be used to constrain $H_{0}$ without using any $\mathrm{CMB}$ measurements. The combination of BAO data and primordial deu-

\footnotetext{
${ }^{1}$ Actually, the DES redshifts in the joint analysis [30] was recalibrated using deep public spectroscopic surveys. Adopting these revised redshifts, the results give a $0.8 \sigma$ reduction in the DESinferred value of $S_{8}$ making the DES results compatible with KiDS and VIKING [25, 26].

${ }^{2}$ The recent TRGB result [39] exhibit somewhat lower agreement with the analysis of gravitationally lensed quasars at the level of $1.4 \sigma$.
} 
terium abundance measurements augmented with supernova distance data [42] or the late-time probe of the matter density [43] leads to the Hubble constraints, in perfect agreement with the Planck measurements. The anisotropic BAO measurements come from galaxy clustering and the Ly $\alpha$ forest along with a precise estimate of the primordial deuterium abundance yield nearly the same Hubble constant [44-46]. It is worth nothing that the recent full-shape power spectrum analyses [47-49] also bring the Hubble measurement into agreement with the Planck data. However, all these measurements are obtained in the context of concordance $\Lambda \mathrm{CDM}$ cosmological model and hence they represent highly model-dependent estimates of $H_{0}$. Given this reason, in our work we focus on the local Cepheid-based distance-ladder measurement [35] which does not rely on early universe physics and being the most widely used local probe to date.

In turn, the ground-based measurements of the CMB anisotropies dictate the estimates consistent with local measurements. Comparison between the Planck and SPT-SZ temperature power spectra reveals that adding the small-scale data from SPT-SZ survey patch drives $2 \sigma$ shifts in various cosmological parameters from the Planck expectation values [50]. In particular, the temperature power spectrum with WMAP7-based Gaussian prior on $\tau$ yields $H_{0}=75.5 \pm 3.5 \mathrm{~km} \mathrm{~s}^{-1} \mathrm{Mpc}^{-1}$ and $\sigma_{8}=$ $0.772 \pm 0.035$ [14]. While the amplitude of density fluctuation $\sigma_{8}$ agrees well with both the Planck results and the direct probes at low redshifts, the $H_{0}$ measurement exhibits more than $2 \sigma$ discrepancy with the Planck value being fully consistent with the distance-ladder estimate [35]. Measurements of CMB polarization power spectra exhibit a similar trend for the $\Lambda \mathrm{CDM}$ parameters to drift away from the Planck values as the SPTPol range is extended to higher multipoles. The SPTPol baseline result reads $H_{0}=71.29 \pm 2.12 \mathrm{~km} \mathrm{~s}^{-1} \mathrm{Mpc}^{-1}$ and $\sigma_{8}=0.771 \pm 0.024$ [17] that reveals a mild preference for lower $\sigma_{8}$ and a $1.8 \sigma$ upward shift in $H_{0}$ from the Planck value.

Above findings demonstrate a perfect consistency of the CMB measurements from the ground with the local probes at low redshifts ${ }^{3}$. On the other hand, the parameter fit based on the ground-based telescopes reveal a distinctive difference with the Planck baseline cosmology. The bulk of this discrepancy is caused by an excess of the lensing-induce smoothing in the Planck maps at small scales. Since the SPT exhibits a higher sensitivity to small scales and it does not detect the excess of lensing power at large multipoles, the idea of replacing the Planck spectrum to that observed by the SPT seems rather natural. Inspired by this simple idea, we present a combined data approach which utilises both Planck and SPT measurements providing consistent parameter inference from both large and small angular scales. For that, we restrict the multipole range of the Planck TT spectrum to $\ell<1000$ and combine

\footnotetext{
${ }^{3}$ The similar concordance between Planck large-scale temperature anisotropies and direct measurements of linear matter density perturbation was obtained in Ref. [51]. They claim 3.7 $\sigma$ tension between the Planck CMB temperature power spectrum at high multipoles $\ell>1000$ and various probes of clustering statistics.
} 
it with the SPTPol measurements of TE and EE power spectra at $50<\ell \leq 8000$. This allows us simultaneously to get rid of the lensing tension, which affects the Planck spectrum at high multipoles, and take benefit from the ground-based experiments where their sensitivity surpass that of the Planck measurements. We proceed in similar fashion to Ref. [52] that combines the CMB damping tail from the partial SPT-SZ survey and seven-year WMAP temperature power spectrum to improve constraints on cosmological parameters. We do not include the TT spectrum at the high multipoles from SPT-SZ survey since this measurement has lower statistical power as compared to the Planck TT spectrum at $\ell<1000$ and does not influence our parameter constraints.

An alternative avenue in handling the tensions between the low and high redshift measurements is a modification of the cosmological concordance model. Indeed, the parameter fit based on the CMB data is highly model dependent and therefore influenced by possible extensions of $\Lambda$ CDM model. Many attempts have been made to restore the concordance between different dataset by modifying either the early or the local Universe physics. The late-time solutions include modified [53-55], interacting [56-60], viscous [61-63] and phenomenologically emergent dark energy models [64, 65], interaction between the dark sectors [66-69] and decaying dark matter [7073]. Recent studies indicate that modification of the Universe expansion history in the two decades of scale factor evolution prior to recombination is needed to solve the $H_{0}$ problem $[74,75]$. The popular early-time solutions are Early Dark Energy (EDE) [76-80] and strong scattering interactions between the neutrinos or between other additional light relics [81-83]. A significant progress in clarifying different discrepancies has been made with cosmology independent reconstruction technique of the Universe evolution [84-86]. For the present status of the Hubble tension one can see [87].

In this paper, we examine possible tensions between the Planck cosmology and astrophysical data with a suitable modification of the early-time cosmology. As a reference, we consider the EDE model. The cornerstone of our analysis is the combined data approach. We discard the Planck temperature power spectrum at high multipoles and combine the remainder with polarization and lensing measurements from SPTPol survey. This strategy eliminates the lensing tension inherent in the Planck spectra on small scales and provides with robust determination of the cosmological parameters. It also allows us to assess the impact of internal inconsistencies within the Planck data on cosmological tensions in the $\Lambda$ CDM and EDE models.

Previous EDE analyses [77, 78, 88] have shown this model can alleviate the Hubble tension, but the late-time amplitude of density fluctuation $\sigma_{8}$ increases as compared to $\Lambda \mathrm{CDM}$, increasing tension with large-scale structure data. Ref. [89] greatly updates previous analyses by considering large-scale structure data in detail. They found that additional weak gravitational lensing and galaxy clustering data substantially weaken the evidence for EDE, as result of the tension between the 
larger values of $S_{8}$ needed to fit the CMB and SH0ES data in the EDE scenario and the lower values of this parameter measured by large-scale structure surveys. In this work, we show that the combined data approach restores concordance amongst these measurements allowing for large $H_{0}$ values without substantially degrading the fit to large-scale structure data. For the first time, we derive EDE constraints from the Planck large-scale CMB anisotropy and the SPTPol data, which are consistent both with SH0ES data and weak lensing measurements along with other direct probes of clustering statistics.

The paper is organized as follows. In Section 2 we introduce all relevant datasets and describe our numerical procedure. In Section 3 we give a detailed description of our analysis method and present parameter constraints in $\Lambda$ CDM model. Section 4 specifies the background and perturbed dynamics of EDE model. In Section 5 we present our final parameter constraints utilizing both the CMB and the low-redshifts measurements within the EDE model. We discuss especially the importance of the intermediate redshift data composed of the supernova dataset and BAO measurements for cosmological inference. Finally, we conclude in Section 6.

\section{Data sets and numerical procedure}

\subsection{Cosmological data}

In our analysis we exploit several different combinations of the following datasets.

- The Planck temperature Plik likelihood truncated at multipoles $30 \leq \ell<1000$ and complemented with Commander power spectrum at low multipoles $\ell<30$ [3]. We include all nuisance parameters and impose the same priors as in the Planck analysis [3] for those. We refer to this likelihood as PlanckTT-low $\ell$.

- The Planck EE likelihood at low multipoles $\ell<30$ [3]. Since the measurement of polarization anisotropies at large scales is essential to constrain the optical depth $\tau$ which breaks the harmful degeneracy between the amplitude of CMB spectra $A_{s}$ and $\tau$ we include this likelihood in all datasets.

- CMB polarization measurements from the 500deg SPTPol survey which includes TE and EE spectra in the multipole range $50<\ell \leq 8000$ [17]. To obtain cosmological constraints we marginalise posteriors over six foreground parameters $\left(D_{3000}^{\mathrm{PS}}, A_{80}^{T T}, A_{80}^{E E}, A_{80}^{T E}, \alpha_{T E}, \alpha_{E E}\right)$, the super-sample lensing variance $\kappa$, two instrumental calibration terms $\left(T_{\text {cal }}, P_{\text {cal }}\right)$ and two beam uncertainty shot noises $\left(A_{\text {beam }}^{1}, A_{\text {beam }}^{2}\right)$. We also impose flat priors on the first four of these and Gaussian priors on the rest in full compliance with [17]. We apply appropriate window functions to transform theoretical spectra from unbinned to binned bandpower space. We denote this likelihood as SPTPol. 
- Measurements of the lensing potential power spectrum $C_{\ell}^{\phi \phi}$ in the multipole range $100<\ell<2000$ from the 500deg SPTPol survey [18]. The lensing potential is reconstructed from a minimum-variance quadratic estimator that combines both the temperature and polarization CMB maps. To incorporate the effects of the survey geometry we convolve the theoretical prediction for $C_{\ell}^{\phi \phi}$ with appropriate window functions at each point in the parameter space. We also perturbatively correct $C_{\ell}^{\phi \phi}$ for changes due to the difference between the recovered lensing spectrum from simulation and the input spectrum following Ref. [19]. We refer to this measurement as Lens in our analysis.

- Combined weak lensing measurements of $S_{8}=0.762 \pm 0.024$ [30] provided by a homogeneous analysis of KiDS, VIKING-450 and DES cosmic shear surveys. Strictly speaking, the full large-scale structure likelihoods can be approximated by a simple Gaussian prior only in the concordance $\Lambda$ CDM model. However, the authors in [89] have justified this procedure for EDE models showing that the information content in large-scale structure data is almost enterily contained in the $S_{8}$ constraint. Guided by this observation, we impose the appropriate Gaussian prior on $S_{8}$ and refer to this measurement as $\mathrm{S}_{8}$.

- The Cepheid-based local measurement of the Hubble constant $H_{0}=74.03 \pm$ $1.42 \mathrm{~km} \mathrm{~s}^{-1} \mathrm{Mpc}^{-1}$ [35]. We implement the SH0ES measurement as the Gaussian prior on $H_{0}$ and call it as $\mathrm{H}_{0}$.

- Baryonic Acoustic Oscillation measurements based on the consensus BOSS DR12 analysis [90] which combines galaxy samples at $z=0.38,0.51$, and 0.61. We name this likelihood BAO.

- Luminosity distances of supernovae Type Ia coming from the Joint Light-curve Analysis (JLA) using SNLS (Supernova Legacy Survey) and SDSS (Sloan Digital Sky Survey) catalogues [91]. We denote this low-redshift probe as SN.

As far as we implemented the SPTPol likelihoods within Montepython environment and the publicly release ${ }^{45}$ was based on another MCMC sampler CosmoMC it makes sense to distribute our code among science community. Our likelihood code is publicly available at https://github.com/ksardase/SPTPol-montepython and can be used for various cosmological analyses.

\section{$2.2 \quad$ Numerical procedure}

All theoretical calculations are carried out in the publicly available Boltzmann CLASS code [92]. We adopt spatially flat Universe and assume normal neutrino hierarchy

\footnotetext{
${ }^{4}$ https://pole.uchicago.edu/public/data/henning17

${ }^{5}$ https://pole.uchicago.edu/public/data/lensing19
} 
pattern with the total active mass $\sum m_{\nu}=0.06 \mathrm{eV}$. To investigate the EDE model we modify background and perturbation equations in CLASS in full compliance with Sec. 4. To recover the posterior distributions in $\Lambda$ CDM and EDE models we apply the Markov chain Monte Carlo (MCMC) approach. For that we use the publicly available MCMC code Montepython [93, 94]. Marginalized posterior densities, limits and contours are produced with the latest version of the getdist package [95] ${ }^{6}$.

To calculate the effects of CMB lensing we use the Halofit suite [96, 97] which allows for modelling the small-scale nonlinear matter power spectrum. These corrections are negligible for the lensed $\mathrm{CMB}$ power spectra but become relevant for the calculation of the lensing potential power spectrum $C_{\ell}^{\phi \phi}$ at high multipoles. We include the Halofit module in all the data procedures.

\section{Methodology and $\Lambda$ CDM constraints}

As a preliminary step, we examine a consistency between PlanckTT-low $\ell$ and SPTPol datasets. For that we calculate posterior distributions for relevant cosmological parameters which are shown in Fig. 1. We reveal that the parameter constraints inferred from PlanckTT-low $\ell$ (blue contours) and SPTPol (red contours) likelihoods are perfectly consistent within $1 \sigma$. Thus, the two likelihoods are consistent with each other, and we proceed to combine them. To quantify the difference between our approach and the standard data procedure we compare posterior distributions for the combined likelihood PlanckTT-low $\ell+$ SPTPol (black contours) and the Planck baseline analysis Planck 2018 (green contours). One may observe that the resulting posteriors deviate from each other at $\lesssim 2 \sigma$. In particular, our combined approach provides substantially lower $S_{8}$ and higher $H_{0}$ as compared to the Planck baseline analysis, thus alleviating the tensions with local measurements.

Parameter constraints inferred from PlanckTT-low $\ell$, SPTPol and its combination are listed in Tab. 1. We found that combining PlanckTT-low $\ell$ and SPTPol datasets one significantly improves constraints on all cosmological parameters except for $\tau$ which mean value and errorbar remain intact. It happens because the corresponding constraint is driven by the CMB polarization measurements on large scales alone which, in turn, is included in all datasets. We stress that the value of $S_{8}$ is now consistent with the homogeneous analysis of cosmic shear surveys [30] and with other direct probes of clustering statistics [20-29]. ThePlanckTT-low $\ell+$ SPTPol dataset also mitigates the Hubble tension to the $2.1 \sigma$ level.

To break the degeneracy between the matter power spectrum $\sigma_{8}$ and the total matter density $\Omega_{m}$ we include measurements of the lensing potential power spectrum. The Lens likelihood is primarily sensitive to the parameter combination $\sigma_{8} \Omega_{m}^{0.25}$ which breaks the degeneracy between $\sigma_{8}$ and $\Omega_{m}$ thus providing a more robust constraint

\footnotetext{
${ }^{6}$ https://github.com/cmbant/getdist
} 


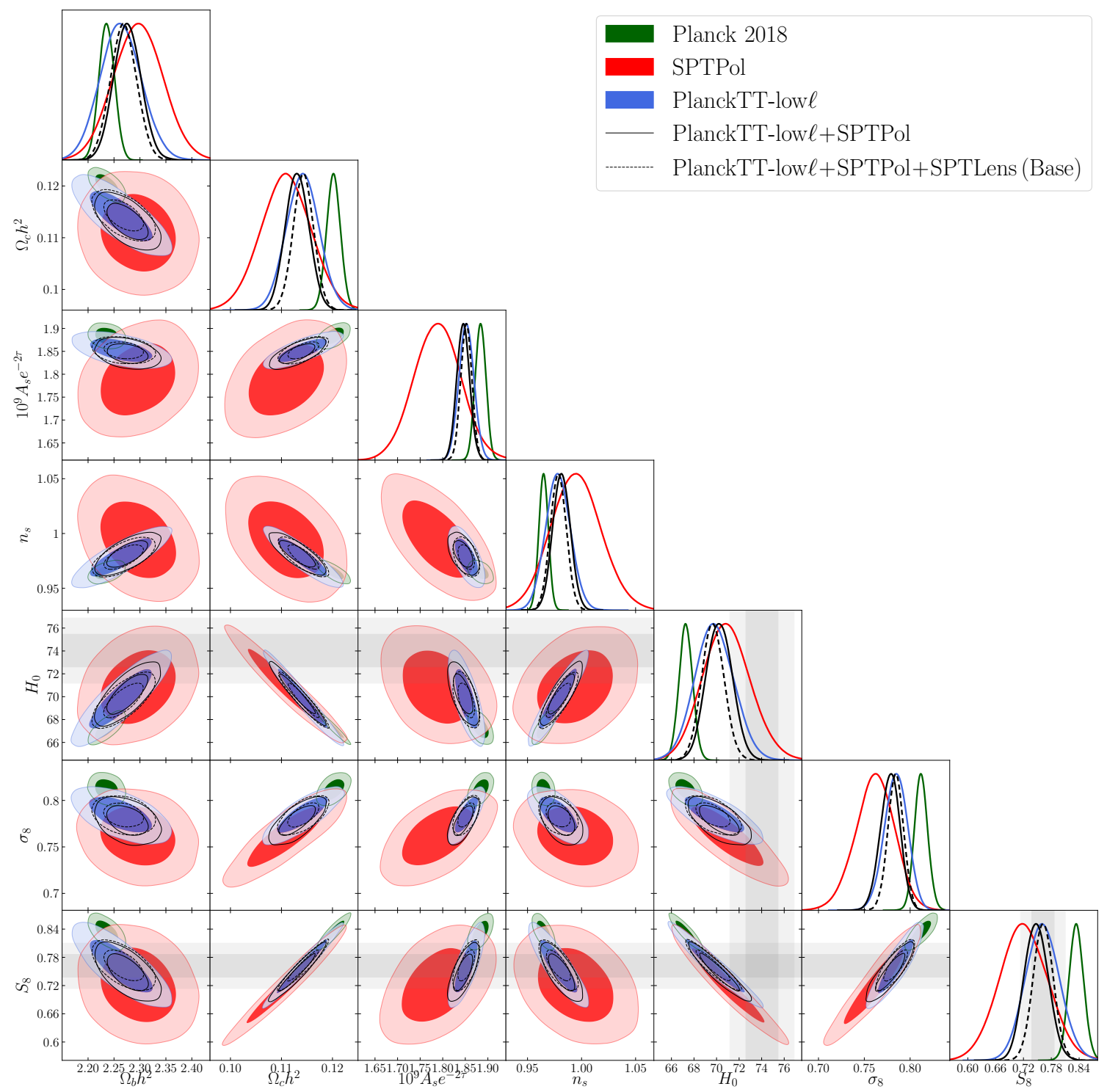

Figure 1. Marginalized parameter constraints for the $\Lambda \mathrm{CDM}$ model using different datasets. We explore independently PlanckTT-low $\ell$ and SPTPol, combined likelihood PlanckTT-low $\ell+$ SPTPol along with SPTPol lensing, PlanckTT-low $\ell+$ SPTPol+Lens. For comparison we include constraints from the baseline Planck analysis PlanckTTTEEE as well. The gray bands represent the $1 \sigma$ and $2 \sigma$ constraints on $S_{8}$ and $H_{0}$ coming from [30] and [35].

on the late time parameter $S_{8}$. The posterior distribution for the PlanckTT-low $\ell+$ SPTPol+Lens dataset is depicted in Fig. 1 (dashed black lines) and the corresponding parameter constraints are tabulated in Tab. 1. We report that adding the Lens likelihood does not induce any significant shifts in parameter constraints except for 


\begin{tabular}{|l|c|c|c|c|}
\hline Parameter & PlanckTT-low $\ell$ & SPTPol & PlanckTT-low $\ell+$ SPTPol & Base \\
\hline \hline $100 \Omega_{b} h^{2}$ & $2.263 \pm 0.040$ & $2.296 \pm 0.048$ & $2.276 \pm 0.026$ & $2.269 \pm 0.025$ \\
$\Omega_{c} h^{2}$ & $0.114 \pm 0.003$ & $0.111 \pm 0.005$ & $0.113 \pm 0.002$ & $0.114 \pm 0.002$ \\
$H_{0}$ & $69.82 \pm 1.72$ & $70.90 \pm 2.12$ & $70.25 \pm 1.15$ & $69.68 \pm 1.00$ \\
$\tau$ & $0.051 \pm 0.009$ & $0.053 \pm 0.010$ & $0.050 \pm 0.009$ & $0.051 \pm 0.009$ \\
$\ln \left(10^{10} A_{s}\right)$ & $3.022 \pm 0.019$ & $2.989 \pm 0.031$ & $3.015 \pm 0.018$ & $3.021 \pm 0.017$ \\
$n_{s}$ & $0.978 \pm 0.012$ & $0.995 \pm 0.023$ & $0.981 \pm 0.008$ & $0.979 \pm 0.007$ \\
\hline$r_{\text {drag }}$ & $145.84 \pm 0.64$ & $146.47 \pm 1.34$ & $146.02 \pm 0.52$ & $145.76 \pm 0.46$ \\
$\Omega_{m}$ & $0.283 \pm 0.020$ & $0.269 \pm 0.026$ & $0.277 \pm 0.013$ & $0.284 \pm 0.012$ \\
$\sigma_{8}$ & $0.784 \pm 0.013$ & $0.762 \pm 0.021$ & $0.778 \pm 0.010$ & $0.784 \pm 0.009$ \\
$S_{8}$ & $0.762 \pm 0.037$ & $0.721 \pm 0.052$ & $0.748 \pm 0.026$ & $0.763 \pm 0.022$ \\
\hline
\end{tabular}

Table 1. Parameter constraints in the standard $\Lambda$ CDM model with $1 \sigma$ errors. Polarization measurements at low multipoles are included in all datasets, see Sec. 2.1. The Base dataset includes PlanckTT-low $\ell+$ SPTPol+Lens.

$S_{8}$ which mean value increases by $0.6 \sigma$ with somewhat reduced error. It occurs because SPTPol TE,EE spectra dictate lower values of the inferred 4-point lensing amplitude with respect to SPTPol lensing measurements [19] which results in higher $S_{8}$ upon adding the Lens likelihood. Our result reveals the remarkable agreement between PlanckTT-low $\ell+$ SPTPol and Lens datasets that justifies the using them in combination. In what follows we refer to the combined likelihood PlanckTT-low $\ell+$ SPTPol+Lens as the Base set. Final constraints from the Base dataset read

$$
S_{8}=0.763 \pm 0.022, \quad H_{0}=69.68 \pm 1.00 \mathrm{~km} \mathrm{~s}^{-1} \mathrm{Mpc}^{-1}
$$

We found that $S_{8}$ value is completely consistent with the local probes, whereas the Hubble tension persists at the $2.5 \sigma$ level. We address this residual tension with one early-time solution in Sec. 5 .

To justify statistical agreement amongst different likelihoods which constitute the Base dataset, we examine the goodness-of-fit to the CMB anisotropies as quantified by the $\chi^{2}$-statistic. In Tab. 2 we list the $\chi^{2}$ values for each CMB likelihood for the best-fit $\Lambda$ CDM model to the baseline Planck 2018 cosmology (second column) and Base dataset (third column). We found that the Base dataset significantly improves $\chi^{2}$-statistic for each likelihood except for the Planck EE $\ell<30$ data with respect to the Planck 2018 baseline analysis. It is caused by internal tensions within Planck data which results in different cosmological inference from low and high multipoles of Planck power spectra. The main culprit of this discrepancy is the overly enhanced lensing smoothing of the CMB peaks which pulls the late-time amplitude $\sigma_{8}$ to a higher value whilst the SPTPol measurements favour lower values of this parameter. Our data procedure is free from this tension and hence harvests a major improvement 


\begin{tabular}{|c|c|c|}
\hline Dataset & Planck 2018 best-fit & Base best-fit \\
\hline \hline Planck TT, $\ell<30$ & 23.41 & 20.93 \\
Planck EE, $\ell<30$ & 396.19 & 396.31 \\
Planck TT, $30 \leq \ell<1000$ & 410.93 & 404.54 \\
SPTPol & 150.04 & 142.52 \\
SPTLens & 7.79 & 5.21 \\
\hline Total $\chi^{2}$ & 988.36 & 969.51 \\
\hline
\end{tabular}

Table 2. $\chi^{2}$ values for the best-fit $\Lambda$ CDM model to the baseline Planck 2018 cosmology and Base dataset.

in the fit to both the Planck TT $\ell<1000$ and SPTPol likelihoods $\left(\Delta \chi_{\mathrm{CMB}}^{2}=-18.85\right)$. Throwing away Planck data at high multipoles gives similar improvement $[3,5,6]$. Meanwhile, we found that the Base dataset notably worsens the $\chi^{2}$-statistic for SPTPol likelihood $\left(\Delta \chi_{\text {SPTPol }}^{2}=3.93\right)$ with respect to a pure SPTPol analysis which gives $\chi_{\text {SPTPol }}^{2}=138.59^{7}$.

\section{1 $A_{L}$ test}

Here we present an important consistency check of cosmological constraints obtained using the combined data approach. Specifically, we verify that the Base dataset provides with a consistent lensing-induced smoothing of acoustic peaks within the $\Lambda$ CDM cosmology. For that, we introduce a free parameter $A_{L}$, which scales the $C_{\ell}^{\phi \phi}$ at each point in the parameter space. Thus scaled lensing potential power spectrum is used to lens the CMB power spectra. Thereby, $A_{L}$ controls the theoretical prediction for the lensing-induced smoothing effect. If the theory is correct and the data is not affected by systematic effects, one expects $A_{L}=1$. In what follows we examine $\Lambda \mathrm{CDM}$ model by making use of the combined data approach.

Varying $A_{L}$ and 6 standard cosmological parameters of the $\Lambda$ CDM concordance model we obtain almost identical posterior distributions with our baseline results outlined in Fig. 1 (dashed black line) and listed in Tab. 1 (Base dataset). We find

$$
A_{L}=0.990 \pm 0.035
$$

This result allows us to conclude that our combined data approach is free from the lensing tension and provides consistent measurements of cosmological parameters in $\Lambda$ CDM model. In general, combining the Planck TT power spectrum in the multipole range $2<\ell<1000$ with the SPTPol measurements of TE and EE spectra at $50<\ell \leq 8000$ enables one to obtain an unbiased cosmological inference from both large and small angular scales.

It is curious to examine the lensing-induced smoothing effect in the PlanckTT-low $\ell+$ SPTPol likelihood without Lens dataset. Following the same strategy we found in

\footnotetext{
${ }^{7} \mathrm{EE}$ polarization measurements at large scales are also considered in this analysis.
} 
this case $A_{L}=0.935 \pm 0.050$. This constraint agrees with the less smoothing of acoustic peaks observed in SPTPol TE and EE spectra [17]. Our result reveals the importance of SPTPol lensing measurements which push the prediction into accordance with the $\Lambda$ CDM model.

\section{Early Dark Energy model}

To handle the residual tensions within $\Lambda \mathrm{CDM}$ model we resort to an early-time solution. For the reference, we consider EDE that behaves like a cosmological constant at early times and then dilutes away with the Universe expansion like radiation or faster at later times. If the dilution starts near the matter-radiation equality it results in a larger Hubble constant and a smaller value of the baryon-photon sound horizon thus alleviating the tensions with the direct cosmological probes. In this Section, we state the homogeneous and perturbed dynamics in the EDE sector pursuing generality and simplicity aims for subsequent implementation in Sec. 5.

First, we introduce one simple realization of EDE in the form of the scalar field. Then, we consistently describe the homogeneous dynamics of EDE field and evolution of its linear perturbations within an effective-fluid approach [98]. We implement all necessary equations in CLASS code and verify that they govern correct background and perturbation dynamics. Finally, we obtain actual constraints on both $\Lambda$ CDM and EDE parameters, examine various tensions and highlight the importance of $\mathrm{BAO}+\mathrm{SN}$ dataset for cosmological inference.

It is worth noting that the effective fluid description is applicable framework to track the scalar field dynamics as advocated in [88]. The previous work [78] claimed the wrong conclusion about the validity of using the approximate fluid approach. Why that study could not fully recover the results of Ref. [77] is easily explained by the different choice of the potential as shown in [88].

\subsection{Background dynamics}

We consider EDE in the form of the cosmological scalar field $\phi$ with a power-law potential

$$
V_{n}(\phi)=V_{0} \frac{\phi^{2 n}}{2^{n}}
$$

where $V_{0}$ denotes the potential amplitude and $n$ states the power-law index. At early times, the Hubble friction dominates and the scalar field undergoes a "slowroll" evolution. During this stage the scalar field is frozen at its initial value $\phi_{i}$ acting as a pure dark energy with equation of state $\omega_{e} \simeq-1$. Once the Hubble parameter drops below a critical value $m$ (which is determined by a form of potential through $m^{2} \simeq \partial^{2} V_{n} / \partial \phi^{2}$ ), the field starts to oscillate around minima of its potential. During the oscillating period the field amplitude decreases in time which dilutes the field energy density. For $n=1$ the field undergoes simple harmonic oscillations with 

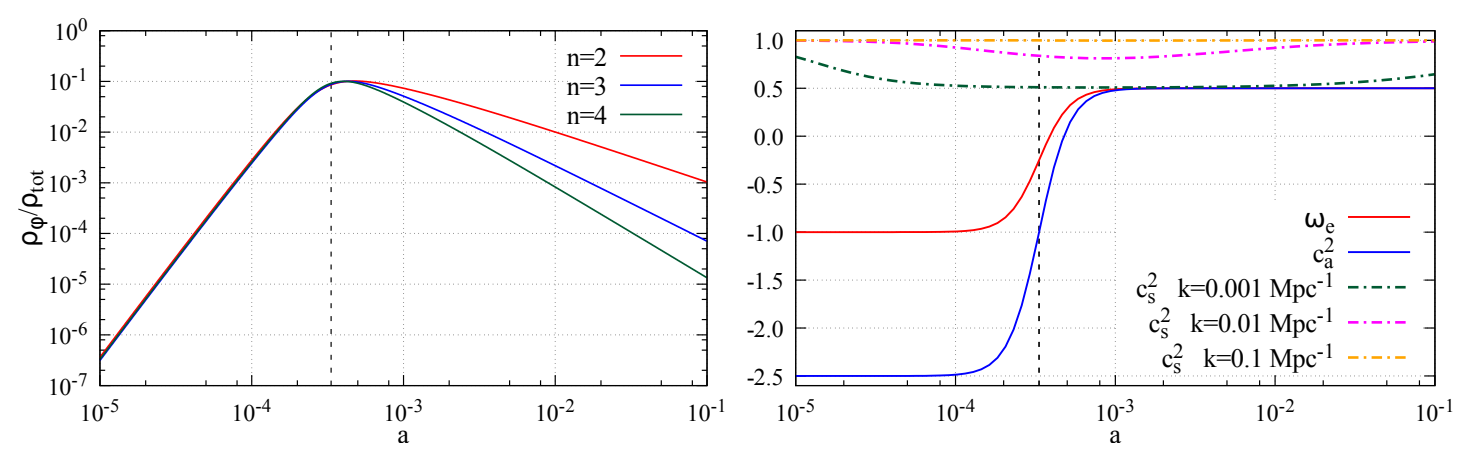

Figure 2. Left panel: evolution of the energy density of the scalar field relative to total energy density of the universe for several values of $n$ 4.1. Right panel: The scalar field equation of state, the adiabatic sound speed $c_{a}^{2}$ and the effective sound speed $c_{s}^{2}$ for various $k$ as a functions of scale factor. Both panels: evolution of all quantities is obtained with $f_{e}=0.1, z_{c}=3000$. The vertical dashed black line refers to $z_{c}=3000$.

a frequency which is independent of its amplitude and for $n>1$ the oscillations are anharmonic and the frequency depends on the amplitude. Rapidly oscillating solutions can be modeled by an effective, averaged-over-cycle description with the effective equation of state $\omega_{e} \simeq \omega_{n}$ given by

$$
\omega_{n}=\frac{n-1}{n+1} .
$$

To provide a smooth transition between the slow-roll and the oscillatory periods we parameterise the energy density of the EDE field averaged over the oscillation period by

$$
\rho_{e}(a)=\frac{2 \rho_{e}\left(a_{c}\right)}{1+\left(a / a_{c}\right)^{3\left(\omega_{n}+1\right)}}
$$

where $a_{c}$ refers to a transition between the two regimes. An associated effective equation of state for the EDE fluid reads

$$
\omega_{e}(a)=\frac{1+\omega_{n}}{1+\left(a_{c} / a\right)^{3\left(\omega_{n}+1\right)}}-1
$$

which asymptotically behaves as $\omega_{e}(a) \rightarrow-1$ at $a \rightarrow 0$ and $\omega_{e}(a) \rightarrow \omega_{n}$ (4.2) for $a \gg a_{c}$. In Fig. 2 we show the evolution of EDE fraction in the total energy density of the Universe averaged over the scalar field oscillations (4.3) for several values of $n$ (4.1) and the effective equation of state of EDE fluid (4.4). On can see that the scalar field contribution to the total energy budget of the Universe dilutes faster for larger $n$.

Ultimately, the background evolution of the EDE field is described by parameters $\rho_{e}\left(a_{c}\right)$ and $a_{c}$. In what follows it is convenient to use the maximum fraction of the 
total energy density in this field $f_{e}$ estimated over the all evolution history, $f_{e}$,

$$
f_{e} \equiv \max \left\{\frac{\rho_{e}}{\rho_{\text {tot }}}\right\}_{\text {all } a}
$$

We note that $f_{e}$ does not necessarily coincide with $\rho_{e}\left(a_{c}\right) / \rho_{\text {tot }}\left(a_{c}\right)$ used in (4.3).

\subsection{Perturbed dynamics}

The effective fluid approach is a powerful tool to describe not only a background evolution but also a perturbed dynamics of the rapidly oscillating scalar field. This formalism provides with a set of approximate 'averaged-over-cycle' equations in interms of fluid variables. In order to utilize the approximate perturbation equations of motion one needs the adiabatic sound speed $c_{a}^{2}$ and the effective sound speed $c_{s}^{2}$. The adiabatic sound speed can be straightforwardly calculated because it depends only on background quantities

$$
c_{a}^{2} \equiv \frac{\dot{P}_{e}}{\dot{\rho}_{e}}=\omega_{e}-\frac{\dot{\omega}_{e}}{3\left(1+\omega_{e}\right) \mathcal{H}}
$$

where $\mathcal{H} \equiv \dot{a} / a$ and the dot refers to the derivative with respect to the conformal time. In Fig. 2 we depict an evolution of the adiabatic sound speed (4.6) as a function of the scale factor. During the slow-roll evolution of the scalar field using (4.4) the adiabatic sound speed equals

$$
c_{a}^{2}=-\frac{3 n+1}{n+1} .
$$

Once the scalar field starts to oscillate, the adiabatic sound speed reaches $\omega_{e}$ (4.4).

In order to compose the approximate perturbation equations we also need the time-averaged effective speed sound in the fluid's rest frame. We adopt the result from [98]

$$
c_{s}^{2} \equiv\left\langle\left\langle\frac{\delta P_{e}}{\delta \rho_{e}}\right\rangle\right\rangle=\frac{2 a^{2}(n-1) \varpi^{2}(a)+k^{2}}{2 a^{2}(n+1) \varpi^{2}(a)+k^{2}},
$$

where $k$ denotes the conformal momentum and $\varpi$ is the instantaneous oscillation frequency of the field fluctuations, which for a pure power law potential is given by [98]

$$
\varpi(a) \simeq \frac{3}{2} H\left(a_{c}\right) \sqrt{\frac{\pi}{n(2 n-1)}} \frac{\Gamma\left(\frac{1+n}{2 n}\right)}{\Gamma\left(\frac{1+2 n}{2 n}\right)} \frac{1}{\left[1+\left(a / a_{c}\right)^{3 /(n+1)}\right]^{n-1}} .
$$

We note that $c_{s}^{2}=1$ for a slowly rolling scalar field but it deviates from 1 once the field starts to oscillate. This feature of models with an early-time energy injection is crucial since $c_{s}^{2}<1$ over a large range of $k$ is needed to resolve the Hubble tension according to Ref. [79].

In the right panel of Fig. 2 we plot the evolution of the effective sound speed (4.8) with the scale factor for three values of $k$. It is worth noting that the standard effective fluid approach assumes an abrupt changing in the relevant quantities, 

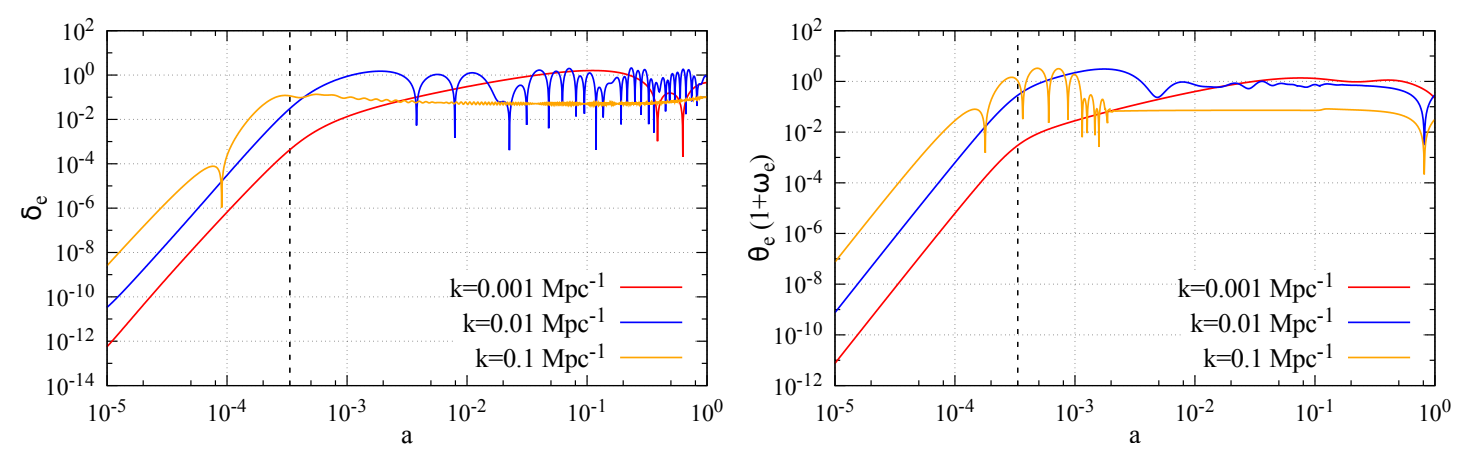

Figure 3. Left panel: the evolution of the density contrast of the scalar field for a set of momentum $k$. Right panel: the evolution of the heat-flux for a set of $k$. Both panels: the evolution of the quantities is obtained for $f_{e}=0.1, z_{c}=3000$. The vertical dashed black line refers to $z_{c}=3000$.

specifically this implies $c_{s}^{2}=1$ and (4.7) at $a<a_{c}$, and (4.8) with (4.6) at $a>a_{c}$. We verify that using the approximate, average-over-cycle quantities, (4.8) and (4.6), rather than the exact ones during all Universe evolution does not lead to significant effects on the predicted power spectra used to constrain the EDE model.

Finally, the equations which govern the evolution of density and velocity perturbations in the synchronous gauge read

$$
\begin{aligned}
& \dot{\delta}_{e}=-\left[u_{e}+\left(1+\omega_{e}\right) \frac{\dot{h}}{2}\right]-3\left(c_{s}^{2}-\omega_{e}\right) \mathcal{H} \delta_{e}-9\left(c_{s}^{2}-c_{a}^{2}\right) \mathcal{H}^{2} \frac{u_{e}}{k^{2}}, \\
& \dot{u}_{e}=-\left(1-3 c_{s}^{2}\right) \mathcal{H} u_{e}+3 \mathcal{H}\left(\omega_{e}-c_{a}^{2}\right) u_{e}+c_{s}^{2} k^{2} \delta_{e},
\end{aligned}
$$

where we introduce the heat-flux $u_{e} \equiv\left(1+\omega_{e}\right) \theta_{e}$ since the linear perturbation equations written in terms of the bulk velocity perturbation $\theta_{e}$ exhibit rapid growth being hard to track numerically. In principle, one should impose adiabatic initial conditions for $\delta_{e}$ and $u_{e}$ on super-Hubble scales. However, the EDE component is always subdominant on superhorizon scales at early times and its perturbations fall inside the gravitational potential created by the radiation component immensely fast. Given this reason, we take $\delta_{e}=u_{e}=0$ initially since these quantities quickly approach a generic solution at radiation dominated stage [99].

In Fig. 3 we depict the evolution of $\delta_{e}$ and $u_{e}$ with the scalar factor for a set of momentum $k$. As long as $c_{s}^{2}=1$, the pressure support leads to a strong decrease in the perturbation amplitude for both superhorizon and subhorizon modes. Once $c_{s}^{2}<1$, see (4.8), the field internal pressure support decreases, that yields nearly constant late-time density and velocity EDE perturbations at $a>a_{c}$, seen in Fig. 3. 


\section{Cosmological constraints within the Early Dark Energy model}

EDE scenario can provide a larger Hubble constant and a smaller value of the baryonphoton sound horizon. While the former trend presents an opportunity to resolve the Hubble tension, the latter feature allows one to reconcile the local measurements of cosmic distance-ladder with the $\mathrm{CMB}$ observations. Indeed, $\mathrm{H}_{0}+\mathrm{BAO}+\mathrm{SN}$ brings a model-independent late-time estimate of the baryon-photon sound horizon at the drag epoch $r_{\text {drag }}$ (decoupling of protons from the cosmic plasma), which is significantly lower than the CMB-inferred value within the $\Lambda$ CDM cosmology $[100,101]$. This mismatch indicates a need for modification of the early-time physics $[74,75]$. In particular, reducing the CMB-inferred sound horizon at the radiation drag epoch by $6-10 \%$ would reconcile the CMB-inferred constraints with the local $H_{0}$ and $r_{\text {drag }}$ determinations. It can be accomplished by an early energy ejection prior to recombination $[76,77]$. In our study we examine this possibility within the EDE framework using the combined data analysis introduced in Sec. 3.

In order to describe the background and perturbed dynamics of the EDE field we modify the CLASS Boltzmann code implementing (4.3), (4.4) and (4.10). We check that these equations produce sensible physical outputs such as power spectra of the EDE fluid. To derive the cosmological constraints within the MCMC approach one needs to specify the parameter space of the EDE model.

The EDE model with a pure power-law potential (4.1) is fully specified by three theory parameters: the redshift $z_{c}$ when the scalar field starts to oscillate, the energy density of the EDE field $\rho_{e}\left(z_{c}\right)$ at $z_{c}$ and the power-law index $n$ which parameterises the potential (4.1). Due to large scatter in $z_{c}$ and $\rho_{e}\left(z_{c}\right)$ variables we consider logarithmic priors on these parameters ${ }^{8}$. We note that absence of the initial scalar field value $\phi_{i}$ in the definition of the effective sound speed parameter (4.8) is caused by the power-law nature of our potential (4.1), see Ref. [88]. Eventually, the parameter space of the EDE model is characterized entirely by $\log _{10}\left(z_{c}\right), \log _{10}\left(\rho_{e}\left(z_{c}\right)\right), n$ and 6 standard parameters $\omega_{b}, \omega_{c}, h, \tau, \ln \left(10^{10} A_{s}\right), n_{s}$. In what follows we consider $n$ as either fixed or free parameter in our fitting procedure.

One comment is in order here. We emphasize that the fitting function calibration implemented in the Halofit module remains valid for EDE cosmology since the models capable of addressing the $H_{0}$ tension require $f_{e} \lesssim 0.1$ which implies a small deviation

\footnotetext{
${ }^{8}$ For the axion-like potential the uniform priors imposed on (physical) particle physics parameters (the axion decay constant and its mass) seriously downweight the preference for EDE models in comparison to uniform priors placed on the effective EDE parameters $\left(f_{e}\right.$ and $\left.\log _{10}\left(z_{c}\right)\right)$ [89]. In fact, this problem is attributed to the search of a proper theoretical solution that would match the prediction of our effective approach. Besides the axion-like potential which does not cover all physical realisations of the EDE scenario, there is a great variety of other EDE setups [80, 102, 103] which have different physical priors. In our analysis, we hold a phenomenological point of view and vary the effective parameters, $f_{e}, \log _{10}\left(z_{c}\right)$, which parameterise the EDE dynamics in a model independent way.
} 
from the $\Lambda \mathrm{CDM}$ expectation. More accurate justification for the validity of using the Halofit module is provided in [89]. Given this reason, we make use of the Halofit module in all EDE analyses.

\subsection{Parameter constraints for $n=3$}

To impose robust constraints on the cosmological parameters, in what follows we fix the shape of energy injection which is determined by parameter $n$, see (4.1). The data seem to favour lower values of the index $n$ since they provide a larger peak energy injection fraction for a fixed width of the transition from slow-roll to oscillatory regime resulting in a larger Hubble constant and smaller value of the baryon-photon sound horizon [78, 88]. On the other hand, lower $n$ models have slowly decaying with the scale factor tail as shown in Fig. 2. Given this reason, it is difficult for these models to inject sufficient energy in a relatively narrow time interval prior to recombination while not having significant residual energy towards low redshifts which, in turn, adversely affects the fit to the CMB data. The fit to cosmological data is thus driven by the two competing effects: injecting the largest possible amount of energy before recombination and dilution of this energy as quickly as possible in the post-recombination era. As a compromise between low and high values of $n$ we choose $n=3$ in our baseline analysis; this choice agrees with the recent EDE studies $[88,89]$. It is worth mentioning that we do not consider $n=2$ which corresponds to the EDE diluting away like radiation: it induces the phenomenon of self-resonance resulting in exponential growth of EDE perturbations [88]. Since we solve only linear perturbation equations for the EDE fluid, our approximate framework can not address the resonance phenomenon properly.

The posterior distributions of the relevant cosmological parameters based on Base $+\mathrm{S}_{8}+\mathrm{H}_{0}$ likelihood are shown in Fig. 4 (red contours). The corresponding parameter constraints are listed in Tab. 3. We found that the Hubble tension is fully resolved in the EDE scenario. Moreover, the fit does not degradate the $S_{8}$ constraint remaining within $1 \sigma$ interval with its local prediction [30]. Eventually, the dataset Base $+\mathrm{S}_{8}+\mathrm{H}_{0}$ imposes $S_{8}=0.762 \pm 0.018$ and $H_{0}=73.06 \pm 1.26 \mathrm{~km} \mathrm{~s}^{-1} \mathrm{Mpc}^{-1}$. Our outcomes are qualitatively similar to results of the previous EDE analyses [78, 88, 89] but, in contrast to them, we claim a perfect consistency with the local measurements owing to the combined data approach introduced in Sec. 3.

A better fit to the local measurements is provided by the early-energy injection just before recombination. Indeed, the marginalized constraint on the redshift transition reads $\log _{10}\left(z_{c}\right)=3.73 \pm 0.20$ which indicates the relatively brief energy injection before recombination. In turn, the maximal injected EDE fraction equals $f_{e}=0.064 \pm 0.025$. We emphasize that a quite brief period of the energy injection around recombination minimizes the impact on other successful $\Lambda \mathrm{CDM}$ predictions hence being essential for the good fit to CMB. The most peculiar background feature in models with early-time energy injection is a positive correlation between $\omega_{c}$ and $f_{e}$ 


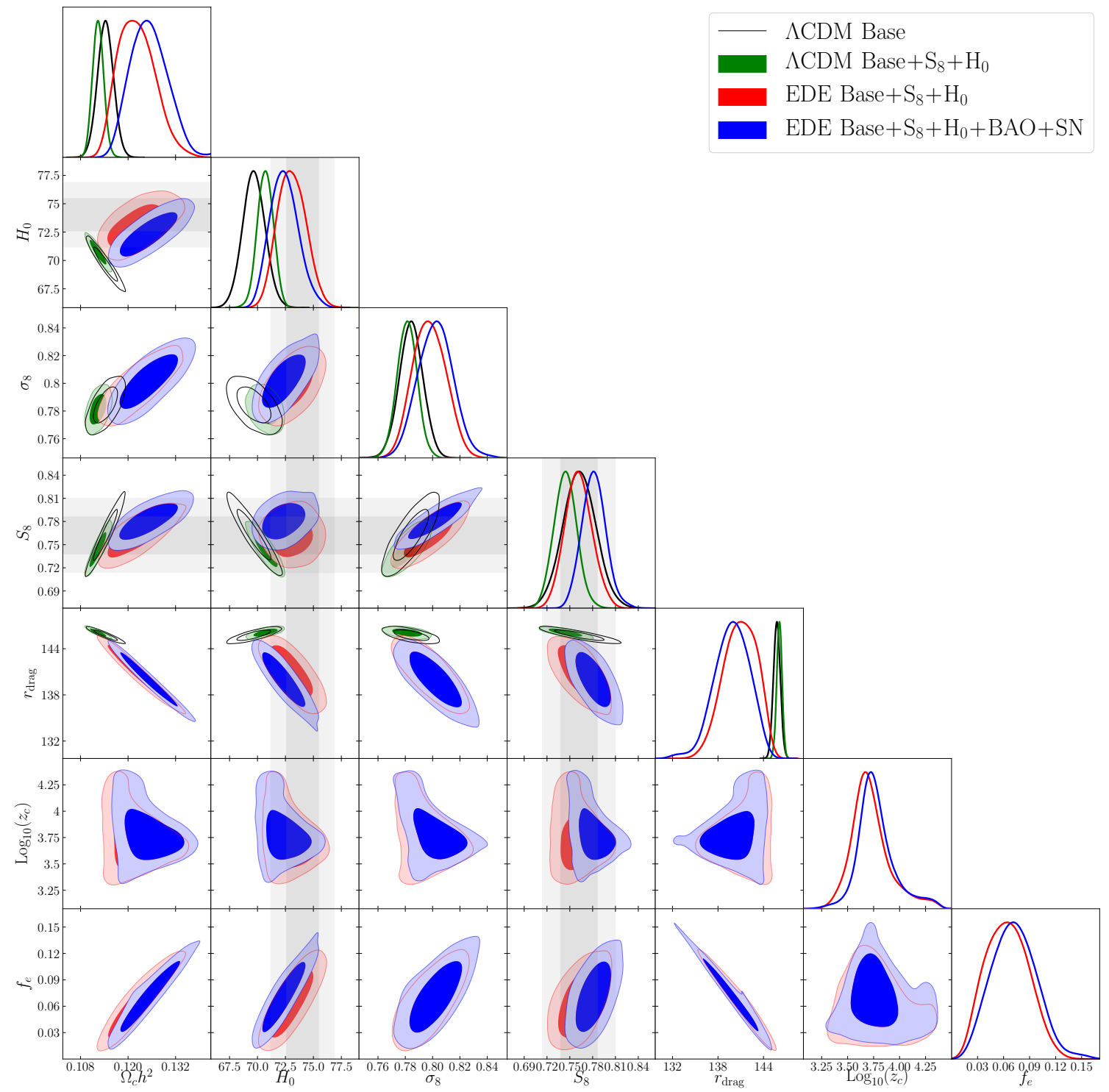

Figure 4. Marginalized parameter constraints for the $\Lambda \mathrm{CDM}$ and EDE models using various datasets. We explore the parameter space using the Base $+\mathrm{S}_{8}+\mathrm{H}_{0}$ dataset with and without intermediate redshift probe $\mathrm{BAO}+\mathrm{SN}$. For comparison we include constraints from the Base data set only in $\Lambda$ CDM model. The Base dataset includes PlanckTT-low $\ell+$ SPTPol+Lens. The gray bands represent the $1 \sigma$ and $2 \sigma$ constraints on $S_{8}$ and $H_{0}$ coming from [30] and [35].

as plotted in Fig. 4. This behaviour is attributed to the early integrated Sachs-Wolf effect which fixes the height of the first CMB temperature acoustic peak. Indeed, the extra energy injected by the oscillating scalar field can be compensated by larger dark matter abundance thereby keeping the Sachs-Wolf amplitude intact. At the 


\begin{tabular}{|l|c|c|c|}
\hline \multirow{2}{*}{ Parameter } & $\Lambda \mathrm{CDM}$ & \multicolumn{2}{|c|}{ EDE } \\
\cline { 2 - 4 } & Base $+\mathrm{S}_{8}+\mathrm{H}_{0}$ & Base $+\mathrm{S}_{8}+\mathrm{H}_{0}$ & Base $+\mathrm{S}_{8}+\mathrm{H}_{0}+\mathrm{BAO}+\mathrm{SN}$ \\
\hline $100 \Omega_{b} h^{2}$ & $2.288 \pm 0.022$ & $2.314 \pm 0.037$ & $2.316 \pm 0.037$ \\
$\Omega_{c} h^{2}$ & $0.112 \pm 0.001$ & $0.122 \pm 0.005$ & $0.126 \pm 0.005$ \\
$H_{0}$ & $70.71 \pm 0.72$ & $73.06 \pm 1.26$ & $72.40 \pm 1.26$ \\
$\tau$ & $0.056 \pm 0.008$ & $0.052 \pm 0.009$ & $0.048 \pm 0.009$ \\
$\ln \left(10^{10} A_{s}\right)$ & $3.027 \pm 0.017$ & $3.032 \pm 0.017$ & $3.027 \pm 0.017$ \\
$n_{s}$ & $0.985 \pm 0.006$ & $0.992 \pm 0.008$ & $0.987 \pm 0.008$ \\
$\log _{10}\left(z_{c}\right)$ & - & $3.73 \pm 0.20$ & $3.78 \pm 0.19$ \\
$\log _{10}\left(\rho_{e}\left(z_{c}\right)\right)$ & - & $2.52 \pm 0.70$ & $2.76 \pm 0.67$ \\
\hline$f_{e}$ & - & $0.064 \pm 0.025$ & $0.072 \pm 0.026$ \\
$r_{\mathrm{drag}}$ & $146.10 \pm 0.35$ & $140.95 \pm 2.19$ & $139.86 \pm 2.35$ \\
$\Omega_{m}$ & $0.272 \pm 0.008$ & $0.274 \pm 0.008$ & $0.285 \pm 0.006$ \\
$\sigma_{8}$ & $0.781 \pm 0.007$ & $0.798 \pm 0.012$ & $0.802 \pm 0.013$ \\
$S_{8}$ & $0.744 \pm 0.015$ & $0.762 \pm 0.018$ & $0.782 \pm 0.016$ \\
\hline
\end{tabular}

Table 3. Parameter constraints in the standard $\Lambda$ CDM and EDE models with $1 \sigma$ errors. Polarization measurements at low multipoles are included to all datasets, see Sec. 2.1. The Base dataset includes PlanckTT-low $\ell+$ SPTPol+Lens.

level of perturbations, the bigger dust component in the early Universe shifts the matter-radiation equality to an earlier epoch. It elongates the matter domination stage and affects the evolution of matter perturbations. In particular, it increases a late-time amplitude of matter fluctuations probed by $\sigma_{8}$. Thus, obtaining a bigger Hubble parameter and a smaller sound horizon at the drag epoch within the models with energy injection near recombination is accompanied by an increase of $\sigma_{8}$ that is shown in Fig. 4. Furthermore, the late-time parameter $S_{8}$ grows as well, that would help to distinguish EDE models from other competing solutions.

To justify the inclusion of Gaussian priors on $S_{8}$ and $H_{0}$ parameters we resort to statistical analysis. The $\chi^{2}$-statistic for each likelihood in the $\Lambda$ CDM and EDE fits to the Base $+\mathrm{S}_{8}+\mathrm{H}_{0}$ dataset is given in Tab. 4. First, it is instructive to compare the $\Lambda \mathrm{CDM}$ fits to $\mathrm{CMB}$ measurements for the Base and Base $+\mathrm{S}_{8}+\mathrm{H}_{0}$ datasets, the $\chi^{2}$ statistic for the former set is provided by Tab. 2 (third column). We found that the goodness-of-fit to CMB data is moderately degraded upon imposing Gaussian priors on $S_{8}$ and $H_{0}\left(\Delta \chi_{\mathrm{CMB}}^{2}=3.3\right)$, predominantly driven by the worsened fit to the Planck TT $30 \leq \ell<1000$ data. This change indicates that the distanceladder Hubble measurement and CMB data are in tension within $\Lambda$ CDM cosmology. Second, we confront the CMB fits to the Base $+\mathrm{S}_{8}+\mathrm{H}_{0}$ likelihood in $\Lambda$ CDM and EDE models. We found a significantly improved CMB fit in the EDE cosmology compared to $\Lambda \mathrm{CDM}\left(\Delta \chi_{\mathrm{CMB}}^{2}=-5.07\right)$, driven primarily by the restored concordance of the 


\begin{tabular}{|c|c|c|}
\hline Dataset & CDM & EDE \\
\hline \hline Planck TT, $\ell<30$ & 20.22 & 20.38 \\
Planck EE, $\ell<30$ & 396.24 & 395.98 \\
Planck TT, $30 \leq \ell<1000$ & 407.00 & 404.61 \\
SPTPol & 143.68 & 142.14 \\
SPTLens & 5.67 & 4.63 \\
$\mathrm{~S}_{8}$ & 0.13 & 0.29 \\
$\mathrm{H}_{0}$ & 6.58 & 0.42 \\
\hline Total $\chi^{2}$ & 979.52 & 968.45 \\
\hline
\end{tabular}

Table 4. $\chi^{2}$ values for the best-fit $\Lambda$ CDM and EDE models to the Base $+\mathrm{S}_{8}+\mathrm{H}_{0}$ dataset.

Planck TT $30 \leq \ell<1000$ likelihood. Furthermore, the EDE model notably improves the $\chi^{2}$-statistic for $\mathrm{CMB}$ data using Base $+\mathrm{S}_{8}+\mathrm{H}_{0}$ dataset with respect to the $\Lambda \mathrm{CDM}$ fit to the Base dataset $\left(\Delta \chi_{\mathrm{CMB}}^{2}=-1.77\right)$. It implies that EDE restores concordance amongst $\mathrm{CMB}$ and SHOES measurements providing even better fit to CMB data in comparison with the $\Lambda \mathrm{CDM}$ model without any priors on $S_{8}$ and $H_{0}$. We emphasize that the EDE model allows for larger $H_{0}$ values without substantially degrading the fit to the cosmic shear measurements. This result justifies the inclusion of additional large-scale structure data within EDE cosmology. It is instructive to compare our results with Ref. [89] that hints at the potential for additional large-scale structure likelihoods to substantially constrain the EDE models. The tight EDE constraints found there arise from the use of the full Planck likelihoods which pull the late-time amplitude $\sigma_{8}$ to higher values $[3,5,6]$. Thus, in order to simultaneously fit the CMB and SH0ES data in the EDE model, one needs even higher values of the latetime amplitude, thereby conflicting with weak lensing and other large-scale structure probes. In contrast, when combining the Planck temperature power spectrum at low multipoles $\ell<1000$ and SPTPol data, we found substantially lower values of $\sigma_{8}$ allowing for the EDE solution of the Hubble tension being consistent with largescale structure data.

An important cross-check of our parameter constraints can be provided by intermediate redshift astrophysical data. The BAO+SN dataset represents a late-time probe of the parameter combination $r_{\text {drag }} h$. An appealing characteristic of this measurement consists in its direct nature since the supernova data allow for translating the cosmic distance scale from the BAO observations to a given redshift in a modelindependent way. Then, one can calibrate the $r_{\text {drag }} h$ measurement with the help of the local Hubble probe [35] to obtain a model-independent determination of the baryon-photon sound horizon at the drag epoch $r_{\text {drag. }}$. On the other hand, the standard ruler $r_{\text {drag }}$ can be obtained through the CMB measurements but this inference strongly depends on the cosmological model assumption. If the late-time probe of $r_{\text {drag }}$ given by $\mathrm{BAO}+\mathrm{SN}+\mathrm{H}_{0}$ matches its early-time estimate within the EDE scenario 


\begin{tabular}{|c|c|c|}
\hline Dataset & CDM & EDE \\
\hline \hline Planck TT, $\ell<30$ & 21.35 & 20.49 \\
Planck EE, $\ell<30$ & 395.66 & 397.02 \\
Planck TT, $30 \leq \ell<1000$ & 405.94 & 405.45 \\
SPTPol & 142.90 & 141.48 \\
SPTLens & 5.37 & 4.47 \\
$\mathrm{~S}_{8}$ & 0.01 & 1.90 \\
$\mathrm{H}_{0}$ & 9.16 & 0.50 \\
$\mathrm{BAO}$ & 7.06 & 4.34 \\
$\mathrm{SN}$ & 683.38 & 683.16 \\
\hline Total $\chi^{2}$ & 1670.83 & 1658.81 \\
\hline
\end{tabular}

Table 5. $\chi^{2}$ values for the best-fit $\Lambda \mathrm{CDM}$ and EDE models to the Base $+\mathrm{S}_{8}+\mathrm{H}_{0}+\mathrm{BAO}+\mathrm{SN}$ data.

provided by the CMB, it will be strong evidence in favour of the EDE model. We examine this possibility by including the $\mathrm{BAO}+\mathrm{SN}$ dataset in what follows.

Resulting constraints from the Base $+\mathrm{S}_{8}+\mathrm{H}_{0}+\mathrm{BAO}+\mathrm{SN}$ dataset are shown in Fig. 4 (blue contours). We observe that the Hubble constant measurement is completely consistent with that from the Base $+\mathrm{S}_{8}+\mathrm{H}_{0}$ dataset, $H_{0}=72.40 \pm 1.26 \mathrm{~km} \mathrm{~s}^{-1} \mathrm{Mpc}^{-1}$. On the contrary, the value of $S_{8}$ underwent substantial raising by $1.1 \sigma$ upwards to $S_{8}=0.782 \pm 0.016$. The further increase of $S_{8}$ is disfavoured by the direct probes of clustering statistics. It means that the upcoming galaxy and weak lensing surveys will elucidate the potential of the EDE fluid to completely reconcile the cosmological tensions.

The main advantage of the intermediate redshift astrophysical data is that it yields the absolute scale for the distance measurements (anchor) at the opposite end of the observable Universe. In particular, $\mathrm{H}_{0}+\mathrm{BAO}+\mathrm{SN}$ provides with a latetime model-independent probe of $r_{\text {drag }}$ which value can be contrasted with the CMB inference under the assumption of the EDE model. We find that inclusion of BAO+SN drives the sound horizon at the drag epoch downwards by $0.5 \sigma$ with the nearly identical error, $r_{\text {drag }}=139.86 \pm 2.35$. This value demonstrates a perfect consistency with the EDE prediction. Reducing of $r_{\text {drag }}$ with respect to the concordance $\Lambda$ CDM model reflects the main property of early-time energy injection models. For instance, the sound horizon at the drag epoch in the EDE scenario is diminished in comparison with the $\Lambda \mathrm{CDM}$ consideration by $4 \%$ for both Base and Base $+\mathrm{S}_{8}+\mathrm{H}_{0}$ datasets that agrees with the previous investigations $[74,101]^{9}$. Regarding the EDE sector, we

\footnotetext{
${ }^{9}$ It worth noting that our constraints on the sound horizon $r_{\text {drag }}$ in the $\Lambda$ CDM cosmology given in Tab. 1 dictate substantially lower values of this parameter with respect to the Planck measurements [3]. It is caused by higher values of $H_{0}$ dictated by our combined data approach which, in turn, results in lower values of $r_{\text {drag }}$. Given this reason, the reduction in sound horizon at drag epoch by
} 

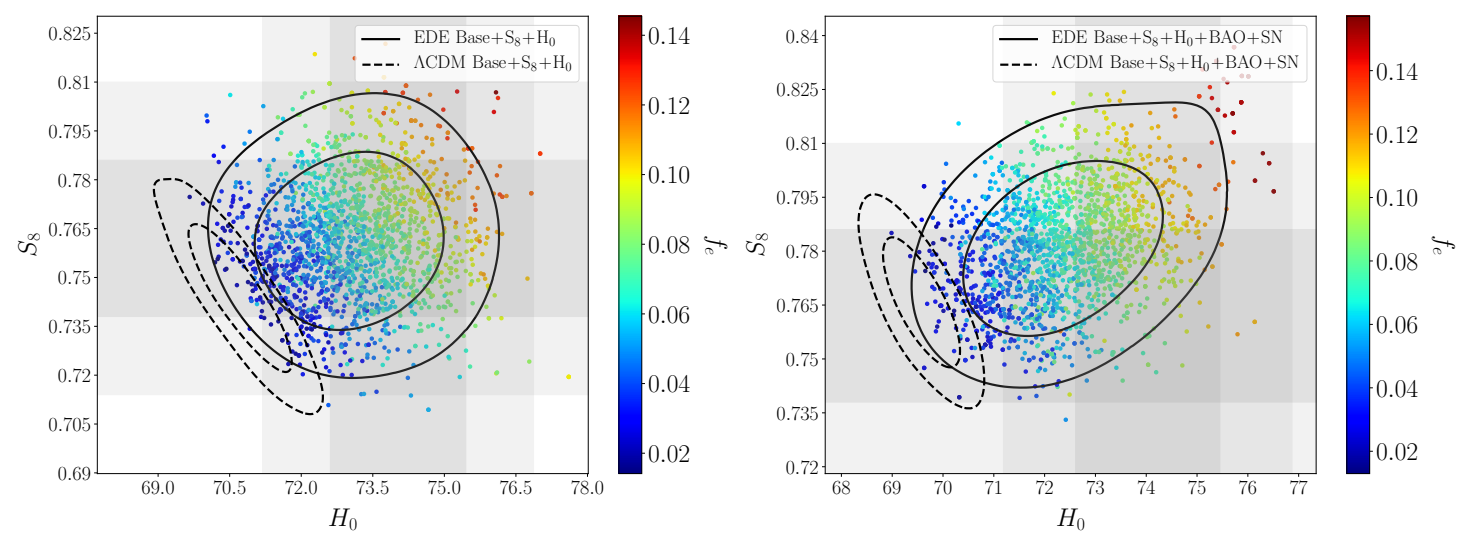

Figure 5. The marginalized posterior distribution in the plane $\left(S_{8}, H_{0}\right)$ for two data set combinations Base $+\mathrm{S}_{8}+\mathrm{H}_{0}$ (left panel) and Base $+\mathrm{S}_{8}+\mathrm{H}_{0}+\mathrm{BAO}+\mathrm{SN}$ (right panel) in the $\Lambda \mathrm{CDM}$ and EDE models. The scattered points represent values of $f_{e}$. The gray bands represent the $1 \sigma$ and $2 \sigma$ constraints on $S_{8}$ and $H_{0}$ coming from [30] and [35]. The Base dataset includes PlanckTT-low $\ell+$ SPTPol+Lens.

obtain $f_{e}=0.072 \pm 0.026$ which indicates a $2.8 \sigma$ evidence for nonzero EDE fraction.

To assess the concordance of $\mathrm{BAO}+\mathrm{SN}$ data with the rest measurements, we provide the $\chi^{2}$-statistic for each likelihood in the $\Lambda$ CDM and EDE fits to the Base+ $\mathrm{S}_{8}+\mathrm{H}_{0}+\mathrm{BAO}+\mathrm{SN}$ dataset in Tab. 5. The $\Lambda \mathrm{CDM}$ fit exhibits a slight tension with the BAO measurements at $1.5 \sigma$ level ${ }^{10}$. This mild tension is completely alleviated in the EDE scenario. The fit to supernova data is not worsened either in the EDE model compared to $\Lambda \mathrm{CDM}$. Regarding the EDE fit to Base $+\mathrm{S}_{8}+\mathrm{H}_{0}$ dataset given in Tab. 4 (third column), we observe nearly the same contributions of all likelihoods to the $\chi^{2}$-statistic except for the cosmic shear measurements which notably degrade the EDE fit $\left(\Delta \chi_{\mathrm{S}_{8}}^{2}=1.61\right)$ highlighting the specific role of future large scale structure data. These results emphasize the remarkable agreement between $\mathrm{BAO}+\mathrm{SN}$ and Base $+\mathrm{S}_{8}+\mathrm{H}_{0}$ likelihoods which justifies the combination of these measurements in one dataset.

Finally, it is instructive to examine the parameter constraints in the plane $\left(S_{8}, H_{0}\right)$ with and without $\mathrm{BAO}+\mathrm{SN}$ dataset. We show the corresponding posterior distributions for EDE and $\Lambda \mathrm{CDM}$ models in Fig. 5. The negative correlation between $S_{8}$ and $H_{0}$ in $\Lambda \mathrm{CDM}$ model can be readily understood. The parameter constraints in this case are mostly driven by the CMB measurements which impose a tight constraint on the observed angular size of the sound horizon at last scattering $\theta_{*}$. For the base $\Lambda \mathrm{CDM}$ model, the main parameter dependence of $\theta_{*}$ is approxi-

$4 \%$ will suffice to reconcile the local Hubble measurements with CMB.

${ }^{10}$ The value $\chi_{\mathrm{BAO}}^{2}=7.06$ is distributed as $\chi_{N}^{2}$ with effective degrees of freedom $N=4$ given by number of data points $\left(D_{A}\right.$ and $H$ at three different redshifts) minus sum of fitting parameters which parametrize the theory prediction $\left(\omega_{c}\right.$ and $\left.h\right)$. 


\begin{tabular}{|c|c|c|c|}
\hline Data set & $\Delta \chi^{2}$ & p-value & Improvement \\
\hline Base $+\mathrm{S}_{8}+\mathrm{H}_{0}$ & 11.1 & 0.003887 & $2.9 \sigma$ \\
Base $+\mathrm{S}_{8}+\mathrm{H}_{0}+\mathrm{BAO}+\mathrm{SN}$ & 12.04 & 0.00243 & $3 \sigma$ \\
\hline Base $+\mathrm{S}_{8}+\mathrm{H}_{0}+\mathrm{BAO}+\mathrm{SN}(n$ free $)$ & 12.04 & 0.007247 & $2.7 \sigma$ \\
\hline
\end{tabular}

Table 6. A statistical improvement of EDE over $\Lambda \mathrm{CDM}$ in fitting the several data sets. In the top panel we set the power-law index to $n=3$ ( 2 extra degrees of freedom) wheres in the bottom line $n$ is treated as a free parameter ( 3 extra degrees of freedom).

mately described by $\omega_{c} \cdot h$ which should be constant. Given this reason, the higher Hubble constant implies lower $\omega_{c}$. Since $\omega_{c}$ is positively correlated with $\sigma_{8}$, the CMB anisotropy in the $\Lambda \mathrm{CDM}$ model yield smaller values of $\Omega_{m}$ and $\sigma_{8}$ thus providing a lower value of $S_{8}$ for higher $H_{0}$, see Fig. 5 (dashed black line). In the EDE case, the degeneracy direction in the plane $\left(S_{8}, H_{0}\right)$ is altered. The reason of that consists in different perturbation dynamics in the EDE model witch results in amplified latetime fluctuations of matter density probed by $\sigma_{8}$. It ensures a negative correlation between $S_{8}$ and $H_{0}$ parameters shown in Fig. 5 (black line). Once we include the intermediate redshift data $\mathrm{BAO}+\mathrm{SN}$, the positive correlation between $S_{8}$ and $H_{0}$ parameters within the EDE scenario becomes more pronounced.

To understand quantitatively which model $(\Lambda \mathrm{CDM}$ or EDE) is preferable, we corroborate our analysis based on posterior distributions with $\chi^{2}$-analysis. For that, we compare the differences in logarithmic likelihoods $\log L$ calculated for these two models in their respective best-fit points for the same datasets. Each difference $\Delta \log L$ is distributed as $\chi^{2}$ with effective degrees of freedom equal to the difference in the number of free parameters in $\Lambda \mathrm{CDM}$ and EDE models. Herein this number equals 2 (recall in this Section we fix $n=3$ ) corresponding to two extra parameters in the EDE model, $\log _{10}\left(\rho_{e}\left(z_{c}\right)\right)$ and $\log _{10}\left(z_{c}\right)$. Resulting improvements are shown in Tab. 6.

Our statistical analysis reveals that the EDE scenario strongly improves the goodness-of-fit by $2.9 \sigma$ compared to $\Lambda$ CDM. The preference does not change if one includes the astrophysical data at intermediate redshifts $\mathrm{BAO}+\mathrm{SN}$ that indicates the remarkable agreement between the early and the late-time cosmological inferences within the EDE model. We argue that the early-time energy injection model provides a much better description of the $\mathrm{CMB}$ and the low-redshift measurements of cosmological parameters.

\subsection{Promoting $n$ to a free parameter}

So far, we have considered $n=3$, based on the cosmological considerations. However, from the phenomenological point of view the shape of power-law potential (4.1) is not known a priory. Hence, it is instructive to explore a broader range of energy injection 

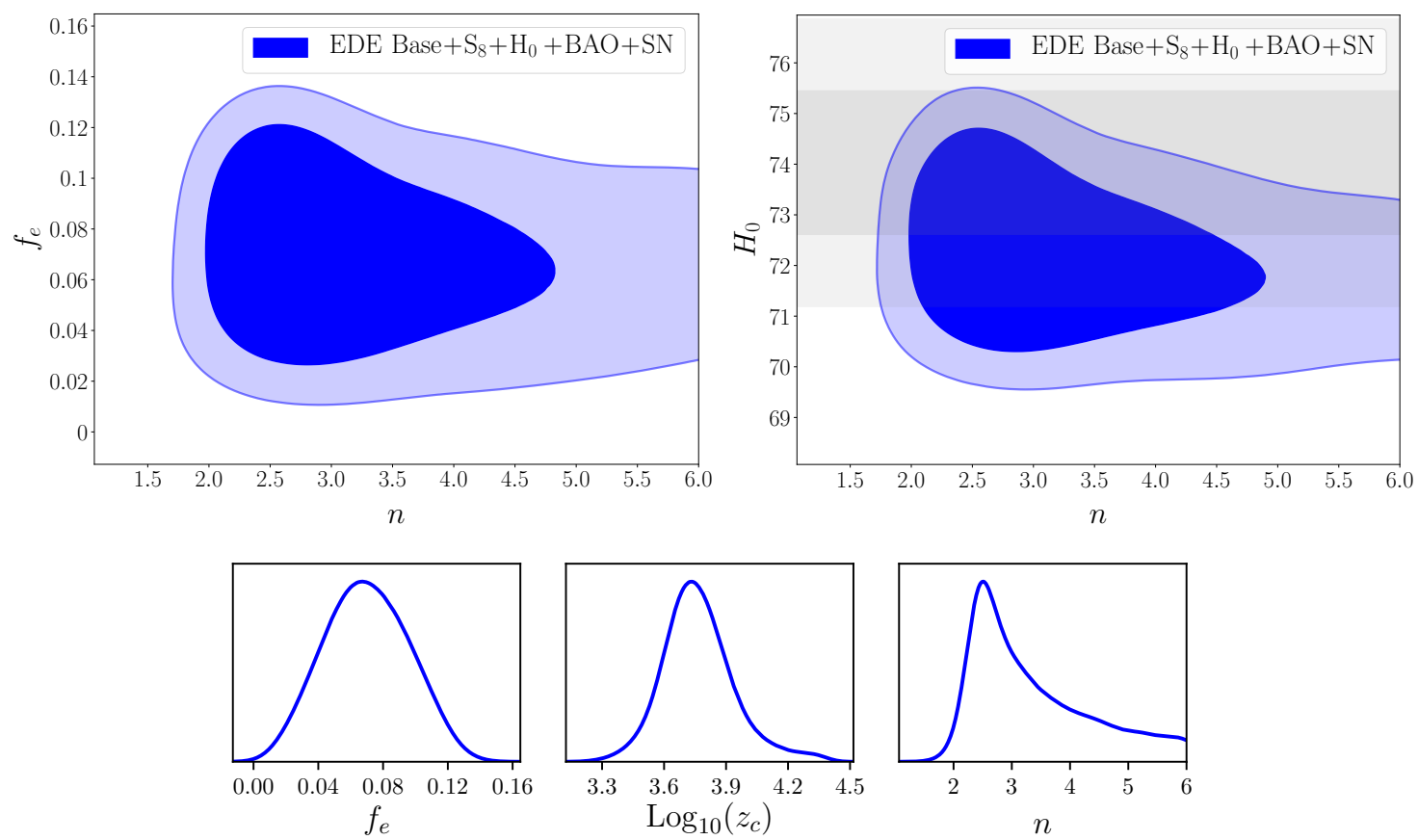

Figure 6. A marginalized posterior distribution in the planes $\left(f_{e}, n\right)$ and $\left(H_{0}, n\right)$ using the Base $+\mathrm{S}_{8}+\mathrm{H}_{0}+\mathrm{BAO}+\mathrm{SN}$ dataset in the EDE model. The gray bands represent the $1 \sigma$ and $2 \sigma$ constraints on $H_{0}$ coming from [35]. The bottom panel represents $1 \mathrm{~d}$ marginalized distributions for $f_{e}, \log _{1010}\left(z_{c}\right)$ and $n$ parameters. The Base dataset includes PlanckTT-low $\ell+$ SPTPol+Lens.

shapes which are controlled by $n$. To this end we allow the power-law index $n$ to float freely in the fit. To choose the appropriate range for $n$ we emphasize that for a significantly large $n$ there is a specific class of no oscillatory (power law) solutions with an asymptotically constant equation of state $[104,105]$. Since the resolution of the Hubble tension requires oscillatory solutions which ensure $c_{s}^{2}<1$ over a large range of $k$ [79], we expect that the region of large $n$ is strongly disfavoured by the data [88]. In our fitting procedure we impose the following flat prior on $n \in[1,6]$.

Our resulting posterior distributions are shown in Fig. 6. As discussed above, the data tends to favor lower values of the index $n$, since they allow for a larger peak energy injection fraction. On the other hand, models with lower $n$ have slowly decaying tail which adversely affect the fit to CMB data. Given this reason, the models with $n<2$ dilute the injected energy too slow and therefore are disfavoured, see the left panel of Fig. 6. The right panel of Fig. 6 clearly illustrates that larger values of the Hubble constant are favoured for $2<n<3$. Our outcome is qualitatively consistent with the previous EDE analyse [78] but extends this study to smaller values of $n$. It has been established owing to effective fluid description which allows for averaging the rapid field oscillations no matter how high the rate is. An explicit numerical 
solution of the field equations is very time consuming exercise that can make the oscillating field dynamics intractable. For instance, computational complexity restricts the previous analysis performed in Ref. [78] to the region $n>2$. It explains why our parameter constraints are somewhat different at $n \sim 2$ as compared to those in [78]. It is worth noting that the existence of the resonance at $n=2$ does not effect our results since the resonance width is too narrow to be captured in our analysis [88]. We verified that the EDE perturbations remain linear and never become comparable to the homogeneous amplitude during the Universe evolution.

We carry out the $\chi^{2}$ statistical analysis for the case of varying power-low index n. Our result is represented in Tab. 6. We do not find any improvement with varying index $n$ compared to the case $n=3$. Somewhat lowering of the overall improvement for the free $n$ analysis is caused by the penalty of adding one additional EDE parameter, i.e. $n$. We confirm that the final result is almost insensitive to the value of $n>2$ supporting the claim of Ref. [77].

\section{Conclusion}

We formulated a new method to analyse the CMB data, that combines the Planck and the SPTPol data in a consistent way. This approach benefits from both fullsky observations and ground-based experiments, and yields an unbiased parameter inference. Using this approach we examine various cosmological tensions in the $\Lambda \mathrm{CDM}$ and the early-time energy injection model. We list our conclusions below.

- $2.5 \sigma$ tension between the CMB constraints and the cosmic shear measurements previously declared in Ref. [30] is completely alleviated in the $\Lambda$ CDM model. It also concerns other local probes of the late-time amplitude of matter density perturbation [20-29]. The upward shift of $\sigma_{8}$ inferred from the Planck analysis is solely driven by an excess of the lensing-induce smoothing of acoustic peaks in the Planck spectra which is absent in our approach. Our resulting constraint on the late-time parameter read $S_{8}=\sigma_{8} \sqrt{\Omega_{m} / 0.3}=0.763 \pm 0.022$.

- Accounting for only the CMB measurements substantially diminishes the Hubble tension with local distance-ladder [35] from $4.4 \sigma$ to $2.5 \sigma$ in the $\Lambda \mathrm{CDM}$ cosmology. The combined fit to the Planck and the SPTPol data drives the $\Lambda \mathrm{CDM}$ fit to the remarkably higher value of the Hubble constant, $H_{0}=$ $69.68 \pm 1.00 \mathrm{~km} \mathrm{~s}^{-1} \mathrm{Mpc}^{-1}$.

- The residual tension with the local distance-ladder measurement of the Hubble constant [35] is completely alleviated in the EDE scenario. Using Base $+\mathrm{S}_{8}+\mathrm{H}_{0}$ dataset we find $H_{0}=73.06 \pm 1.26 \mathrm{~km} \mathrm{~s}^{-1} \mathrm{Mpc}^{-1}$. At the same time, it does not degrade the fit to the direct probe of the late-time amplitude [30] leading to 
$S_{8}=\sigma_{8} \sqrt{\Omega_{m} / 0.3}=0.762 \pm 0.018$. It relieves the conflict between the SH0ESresolving EDE cosmologies and large-scale structure data recently claimed in Ref. [89].

- The intermediate redshifts data BAO+SN present important consistency check of the EDE model since it provides an independent probe of $r_{\text {drag }} h$ at late times. We found that the parameter constraints driven by $\mathrm{H}_{0}+\mathrm{BAO}+\mathrm{SN}$ are in excellent agreement with the EDE prediction. In particular, the Base $+\mathrm{S}_{8}+\mathrm{H}_{0}+$ $\mathrm{BAO}+\mathrm{SN}$ dataset yields somewhat lower value of the Hubble constant $H_{0}=$ $72.40 \pm 1.26 \mathrm{~km} \mathrm{~s}^{-1} \mathrm{Mpc}^{-1}$. At the same time, it results in a substantially higher amplitude of late-time matter perturbation characterized by $S_{8}=0.782 \pm 0.016$, but remaining within $1 \sigma$ interval with its local measurement [30].

- The cosmological data disfavour the early-time injection models with the powerlaw indexes $n<2$. Furthermore, setting free the power-law index $n$ we do not find any improvement in comparison with the case of $n=3$.

We emphasize that ongoing and future galaxy probes such as DESI, Euclid and LSST will clarify the potential of early-time energy injection model to completely alleviate various cosmological tensions.

\section{Acknowledgments}

We thank Mikhail Ivanov for helpful discussions. We also thank Vivian Poulin for valuable comments. The work was supported by the RSF grant 17-12-01547. All numerical calculations were performed on the Computational Cluster of the Theoretical division of INR RAS and the MVS-10P supercomputer of the Joint Supercomputer Center of the Russian Academy of Sciences (JSCC RAS).

\section{References}

[1] A. Lewis and A. Challinor, Weak gravitational lensing of the CMB, Phys. Rept. 429 (2006) 1 [astro-ph/0601594].

[2] R. Sunyaev and Y. Zeldovich, Small scale fluctuations of relic radiation, Astrophys. Space Sci. 7 (1970) 3.

[3] Planck collaboration, N. Aghanim et al., Planck 2018 results. VI. Cosmological parameters, 1807.06209.

[4] Planck collaboration, P. A. R. Ade et al., Planck 2015 results. XIII. Cosmological parameters, Astron. Astrophys. 594 (2016) A13 [1502.01589].

[5] G. E. Addison, Y. Huang, D. J. Watts, C. L. Bennett, M. Halpern, G. Hinshaw et al., Quantifying discordance in the 2015 Planck CMB spectrum, Astrophys. J. 818 (2016) 132 [1511.00055]. 
[6] Planck collaboration, N. Aghanim et al., Planck intermediate results. LI. Features in the cosmic microwave background temperature power spectrum and shifts in cosmological parameters, Astron. Astrophys. 607 (2017) A95 [1608.02487].

[7] P. Motloch and W. Hu, Lensing-like tensions in the Planck legacy release, 1912.06601.

[8] M. Lueker et al., Measurements of Secondary Cosmic Microwave Background Anisotropies with the South Pole Telescope, Astrophys. J. 719 (2010) 1045 [0912.4317].

[9] E. Shirokoff et al., Improved constraints on cosmic microwave background secondary anisotropies from the complete 2008 South Pole Telescope data, Astrophys. J. 736 (2011) 61 [1012.4788].

[10] C. Reichardt et al., A measurement of secondary cosmic microwave background anisotropies with two years of South Pole Telescope observations, Astrophys. J. 755 (2012) 70 [1111.0932].

[11] ACT collaboration, J. Fowler et al., The Atacama Cosmology Telescope: A Measurement of the $600<$ ell $<8000$ Cosmic Microwave Background Power Spectrum at 148 GHz, Astrophys. J. 722 (2010) 1148 [1001.2934].

[12] S. Das et al., The Atacama Cosmology Telescope: A Measurement of the Cosmic Microwave Background Power Spectrum at 148 and $218 \mathrm{GHz}$ from the 2008 Southern Survey, Astrophys. J. 729 (2011) 62 [1009.0847].

[13] Polarbear collaboration, S. Adachi et al., A measurement of the CMB E-mode angular power spectrum at subdegree scales from 670 square degrees of POLARBEAR data, 2005.06168.

[14] K. T. Story et al., A Measurement of the Cosmic Microwave Background Damping Tail from the 2500-square-degree SPT-SZ survey, Astrophys. J. 779 (2013) 86 [1210.7231].

[15] M. Seiffert, C. Borys, D. Scott and M. Halpern, An upper limit to polarized submillimetre emission in Arp 220, Mon. Not. Roy. Astron. Soc. 374 (2007) 409 [astro-ph/0610485].

[16] R. Battye, I. Browne, M. Peel, N. Jackson and C. Dickinson, Statistical properties of polarized radio sources at high frequency and their impact on CMB polarization measurements, Mon. Not. Roy. Astron. Soc. 413 (2011) 132 [1003.5846].

[17] SPT collaboration, J. W. Henning et al., Measurements of the Temperature and E-Mode Polarization of the CMB from 500 Square Degrees of SPTpol Data, Astrophys. J. 852 (2018) 97 [1707.09353].

[18] W. L. K. Wu et al., A Measurement of the Cosmic Microwave Background Lensing Potential and Power Spectrum from $500 \mathrm{deg}^{2}$ of SPTpol Temperature and Polarization Data, Astrophys. J. 884 (2019) 70 [1905.05777].

[19] SPT collaboration, F. Bianchini et al., Constraints on Cosmological Parameters 
from the $500 \mathrm{deg}^{2}$ SPTpol Lensing Power Spectrum, 1910.07157.

[20] SPT collaboration, S. Bocquet et al., Cluster Cosmology Constraints from the 2500 $\mathrm{deg}^{2}$ SPT-SZ Survey: Inclusion of Weak Gravitational Lensing Data from Magellan and the Hubble Space Telescope, Astrophys. J. 878 (2019) 55 [1812.01679].

[21] Planck collaboration, P. A. R. Ade et al., Planck 2015 results. XXIV. Cosmology from Sunyaev-Zeldovich cluster counts, Astron. Astrophys. 594 (2016) A24 [1502.01597].

[22] A. Vikhlinin et al., Chandra Cluster Cosmology Project III: Cosmological Parameter Constraints, Astrophys. J. 692 (2009) 1060 [0812.2720].

[23] H. Böhringer, G. Chon and C. A. Collins, The extended ROSAT-ESO Flux Limited X-ray Galaxy Cluster Survey (REFLEX II) IV. X-ray Luminosity Function and First Constraints on Cosmological Parameters, Astron. Astrophys. 570 (2014) A31 [1403.2927].

[24] H. Böhringer, G. Chon, J. Retzlaff, J. Trümper, K. Meisenheimer and N. Schartel, The extended Northern ROSAT Galaxy Cluster Survey (NORAS II) I. Survey Construction and First Results, Astron. J. 153 (2017) 220 [1704.06489].

[25] H. Hildebrandt et al., KiDS-450: Cosmological parameter constraints from tomographic weak gravitational lensing, Mon. Not. Roy. Astron. Soc. 465 (2017) 1454 [1606.05338].

[26] H. Hildebrandt et al., KiDS+VIKING-450: Cosmic shear tomography with optical+infrared data, Astron. Astrophys. 633 (2020) A69 [1812.06076].

[27] DES collaboration, T. M. C. Abbott et al., Dark Energy Survey year 1 results: Cosmological constraints from galaxy clustering and weak lensing, Phys. Rev. D98 (2018) 043526 [1708.01530].

[28] DES, SPT collaboration, T. M. C. Abbott et al., Dark Energy Survey Year 1 Results: Joint Analysis of Galaxy Clustering, Galaxy Lensing, and CMB Lensing Two-point Functions, Phys. Rev. D100 (2019) 023541 [1810.02322].

[29] HSC collaboration, C. Hikage et al., Cosmology from cosmic shear power spectra with Subaru Hyper Suprime-Cam first-year data, Publ. Astron. Soc. Jap. 71 (2019) Publications of the Astronomical Society of Japan, Volume 71, Issue 2, April 2019, 43, https://doi.org/10.1093/pasj/psz010 [1809. 09148].

[30] S. Joudaki et al., KiDS+VIKING-450 and DES-Y1 combined: Cosmology with cosmic shear, 1906.09262.

[31] M. Asgari et al., KiDS+VIKING-450 and DES-Y1 combined: Mitigating baryon feedback uncertainty with COSEBIs, 1910.05336.

[32] HST collaboration, W. L. Freedman et al., Final results from the Hubble Space Telescope key project to measure the Hubble constant, Astrophys. J. 553 (2001) 47 [astro-ph/0012376]. 
[33] W. L. Freedman, B. F. Madore, V. Scowcroft, C. Burns, A. Monson, S. E. Persson et al., Carnegie Hubble Program: A Mid-Infrared Calibration of the Hubble Constant, Astrophys. J. 758 (2012) 24 [1208.3281].

[34] A. G. Riess et al., A 2.4\% Determination of the Local Value of the Hubble Constant, Astrophys. J. 826 (2016) 56 [1604.01424].

[35] A. G. Riess, S. Casertano, W. Yuan, L. M. Macri and D. Scolnic, Large Magellanic Cloud Cepheid Standards Provide a 1\% Foundation for the Determination of the Hubble Constant and Stronger Evidence for Physics beyond $\Lambda$ CDM, Astrophys. J. 876 (2019) 85 [1903.07603].

[36] D. W. Pesce et al., The Megamaser Cosmology Project. XIII. Combined Hubble constant constraints, Astrophys. J. 891 (2020) L1 [2001.09213].

[37] C. D. Huang, A. G. Riess, W. Yuan, L. M. Macri, N. L. Zakamska, S. Casertano et al., Hubble Space Telescope Observations of Mira Variables in the Type Ia Supernova Host NGC 1559: An Alternative Candle to Measure the Hubble Constant, 1908.10883.

[38] S. Taubenberger, S. H. Suyu, E. Komatsu, I. Jee, S. Birrer, V. Bonvin et al., The Hubble Constant determined through an inverse distance ladder including quasar time delays and Type Ia supernovae, Astron. Astrophys. 628 (2019) L7 [1905.12496].

[39] W. L. Freedman, B. F. Madore, T. Hoyt, I. S. Jang, R. Beaton, M. G. Lee et al., Calibration of the Tip of the Red Giant Branch (TRGB), 2002.01550.

[40] K. C. Wong et al., HOLiCOW XIII. A 2.4\% measurement of $H_{0}$ from lensed quasars: $5.3 \sigma$ tension between early and late-Universe probes, 1907.04869.

[41] K. Liao, A. Shafieloo, R. E. Keeley and E. V. Linder, Determining $H_{0}$ Model-Independently and Consistency Tests, 2002.10605.

[42] P. Lemos, E. Lee, G. Efstathiou and S. Gratton, Model independent H(z) reconstruction using the cosmic inverse distance ladder, Mon. Not. Roy. Astron. Soc. 483 (2019) 4803 [1806.06781].

[43] DES collaboration, T. Abbott et al., Dark Energy Survey Year 1 Results: A Precise HO Estimate from DES Y1, BAO, and D/H Data, Mon. Not. Roy. Astron. Soc. 480 (2018) 3879 [1711.00403].

[44] N. Schöneberg, J. Lesgourgues and D. C. Hooper, The BAO+BBN take on the Hubble tension, JCAP 10 (2019) 029 [1907.11594].

[45] A. Cuceu, J. Farr, P. Lemos and A. Font-Ribera, Baryon Acoustic Oscillations and the Hubble Constant: Past, Present and Future, JCAP 10 (2019) 044 [1906.11628].

[46] G. Addison, D. Watts, C. Bennett, M. Halpern, G. Hinshaw and J. Weiland, Elucidating $\Lambda$ CDM: Impact of Baryon Acoustic Oscillation Measurements on the Hubble Constant Discrepancy, Astrophys. J. 853 (2018) 119 [1707.06547]. 
[47] M. M. Ivanov, M. Simonović and M. Zaldarriaga, Cosmological Parameters from the BOSS Galaxy Power Spectrum, 1909.05277.

[48] G. D’Amico, J. Gleyzes, N. Kokron, D. Markovic, L. Senatore, P. Zhang et al., The Cosmological Analysis of the SDSS/BOSS data from the Effective Field Theory of Large-Scale Structure, 1909.05271.

[49] T. Tröster et al., Cosmology from large-scale structure: Constraining $\Lambda$ CDM with BOSS, Astron. Astrophys. 633 (2020) L10 [1909.11006].

[50] SPT collaboration, K. Aylor et al., A Comparison of Cosmological Parameters Determined from CMB Temperature Power Spectra from the South Pole Telescope and the Planck Satellite, Astrophys. J. 850 (2017) 101 [1706.10286].

[51] R. A. Burenin, Measurements of the Matter Density Perturbation Amplitude from Cosmological Data, Astron. Lett. 44 (2018) 653 [1806.03261].

[52] R. Keisler et al., A Measurement of the Damping Tail of the Cosmic Microwave Background Power Spectrum with the South Pole Telescope, Astrophys. J. 743 (2011) 28 [1105.3182].

[53] E. Di Valentino, A. Melchiorri, E. V. Linder and J. Silk, Constraining Dark Energy Dynamics in Extended Parameter Space, Phys. Rev. D96 (2017) 023523 [1704.00762].

[54] Q.-G. Huang and K. Wang, How the dark energy can reconcile Planck with local determination of the Hubble constant, Eur. Phys. J. C76 (2016) 506 [1606.05965].

[55] M.-M. Zhao, D.-Z. He, J.-F. Zhang and X. Zhang, Search for sterile neutrinos in holographic dark energy cosmology: Reconciling Planck observation with the local measurement of the Hubble constant, Phys. Rev. D96 (2017) 043520 [1703.08456].

[56] E. Di Valentino, A. Melchiorri and J. Silk, Reconciling Planck with the local value of $H_{0}$ in extended parameter space, Phys. Lett. B761 (2016) 242 [1606.00634].

[57] E. Di Valentino, A. Melchiorri and O. Mena, Can interacting dark energy solve the $H_{0}$ tension?, Phys. Rev. D96 (2017) 043503 [1704.08342].

[58] E. Di Valentino, A. Melchiorri, O. Mena and S. Vagnozzi, Interacting dark energy after the latest Planck, DES, and $H_{0}$ measurements: an excellent solution to the $H_{0}$ and cosmic shear tensions, 1908.04281.

[59] E. Di Valentino, A. Melchiorri, O. Mena and S. Vagnozzi, Non-minimal dark sector physics and cosmological tensions, 1910.09853.

[60] S. Kumar, R. C. Nunes and S. K. Yadav, Dark sector interaction: a remedy of the tensions between CMB and LSS data, Eur. Phys. J. C79 (2019) 576 [1903.04865].

[61] B. Mostaghel, H. Moshafi and S. M. S. Movahed, Non-minimal Derivative Coupling Scalar Field and Bulk Viscous Dark Energy, Eur. Phys. J. C77 (2017) 541 [1611.08196].

[62] D. Wang, Y.-J. Yan and X.-H. Meng, Constraining viscous dark energy models with 
the latest cosmological data, Eur. Phys. J. C77 (2017) 660.

[63] W. Yang, S. Pan, E. Di Valentino, A. Paliathanasis and J. Lu, Challenging bulk viscous unified scenarios with cosmological observations, Phys. Rev. D100 (2019) 103518 [1906.04162].

[64] X. Li and A. Shafieloo, A Simple Phenomenological Emergent Dark Energy Model can Resolve the Hubble Tension, Astrophys. J. 883 (2019) L3 [1906.08275].

[65] S. Pan, W. Yang, E. Di Valentino, A. Shafieloo and S. Chakraborty, Reconciling $H_{0}$ tension in a six parameter space?, 1907.12551.

[66] P. Ko and Y. Tang, Light dark photon and fermionic dark radiation for the Hubble constant and the structure formation, Phys. Lett. B762 (2016) 462 [1608.01083].

[67] M. Raveri, W. Hu, T. Hoffman and L.-T. Wang, Partially Acoustic Dark Matter Cosmology and Cosmological Constraints, Phys. Rev. D96 (2017) 103501 [1709.04877].

[68] E. Di Valentino, C. Bøehm, E. Hivon and F. R. Bouchet, Reducing the $H_{0}$ and $\sigma_{8}$ tensions with Dark Matter-neutrino interactions, Phys. Rev. D97 (2018) 043513 [1710.02559].

[69] M. Archidiacono, D. C. Hooper, R. Murgia, S. Bohr, J. Lesgourgues and M. Viel, Constraining Dark Matter-Dark Radiation interactions with $C M B, B A O$, and

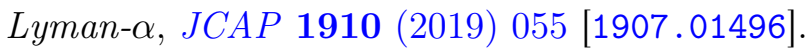

[70] Z. Berezhiani, A. D. Dolgov and I. I. Tkachev, Reconciling Planck results with low redshift astronomical measurements, Phys. Rev. D92 (2015) 061303 [1505.03644].

[71] A. Chudaykin, D. Gorbunov and I. Tkachev, Dark matter component decaying after recombination: Lensing constraints with Planck data, Phys. Rev. D94 (2016) 023528 [1602.08121].

[72] V. Poulin, P. D. Serpico and J. Lesgourgues, A fresh look at linear cosmological constraints on a decaying dark matter component, JCAP 1608 (2016) 036 [1606.02073].

[73] A. Chudaykin, D. Gorbunov and I. Tkachev, Dark matter component decaying after recombination: Sensitivity to baryon acoustic oscillation and redshift space distortion probes, Phys. Rev. D97 (2018) 083508 [1711.06738].

[74] J. L. Bernal, L. Verde and A. G. Riess, The trouble with $H_{0}$, JCAP 1610 (2016) 019 [1607.05617].

[75] K. Aylor, M. Joy, L. Knox, M. Millea, S. Raghunathan and W. L. K. Wu, Sounds Discordant: Classical Distance Ladder $\& 4$ CDM -based Determinations of the Cosmological Sound Horizon, Astrophys. J. 874 (2019) 4 [1811.00537].

[76] T. Karwal and M. Kamionkowski, Dark energy at early times, the Hubble parameter, and the string axiverse, Phys. Rev. D94 (2016) 103523 [1608.01309].

[77] V. Poulin, T. L. Smith, T. Karwal and M. Kamionkowski, Early Dark Energy Can 
Resolve The Hubble Tension, Phys. Rev. Lett. 122 (2019) 221301 [1811.04083].

[78] P. Agrawal, F.-Y. Cyr-Racine, D. Pinner and L. Randall, Rock 'n' Roll Solutions to the Hubble Tension, 1904.01016.

[79] M.-X. Lin, G. Benevento, W. Hu and M. Raveri, Acoustic Dark Energy: Potential Conversion of the Hubble Tension, Phys. Rev. D100 (2019) 063542 [1905.12618].

[80] G. Ye and Y.-S. Piao, Is the Hubble tension a hint of AdS around recombination?, 2001.02451.

[81] F.-Y. Cyr-Racine and K. Sigurdson, Limits on Neutrino-Neutrino Scattering in the Early Universe, Phys. Rev. D90 (2014) 123533 [1306.1536].

[82] L. Lancaster, F.-Y. Cyr-Racine, L. Knox and Z. Pan, A tale of two modes: Neutrino free-streaming in the early universe, JCAP 1707 (2017) 033 [1704.06657].

[83] C. D. Kreisch, F.-Y. Cyr-Racine and O. Doré, The Neutrino Puzzle: Anomalies, Interactions, and Cosmological Tensions, 1902.00534.

[84] S. Joudaki, M. Kaplinghat, R. Keeley and D. Kirkby, Model independent inference of the expansion history and implications for the growth of structure, Phys. Rev. D97 (2018) 123501 [1710.04236].

[85] V. Poulin, K. K. Boddy, S. Bird and M. Kamionkowski, Implications of an extended dark energy cosmology with massive neutrinos for cosmological tensions, Phys. Rev. D97 (2018) 123504 [1803.02474].

[86] R. E. Keeley, S. Joudaki, M. Kaplinghat and D. Kirkby, Implications of a transition in the dark energy equation of state for the $H_{0}$ and $\sigma_{8}$ tensions, JCAP 1912 (2019) 035 [1905.10198].

[87] L. Knox and M. Millea, The Hubble Hunter's Guide, 1908.03663.

[88] T. L. Smith, V. Poulin and M. A. Amin, Oscillating scalar fields and the Hubble tension: a resolution with novel signatures, 1908.06995.

[89] J. C. Hill, E. McDonough, M. W. Toomey and S. Alexander, Early Dark Energy Does Not Restore Cosmological Concordance, 2003.07355.

[90] BOSS collaboration, S. Alam et al., The clustering of galaxies in the completed SDSS-III Baryon Oscillation Spectroscopic Survey: cosmological analysis of the DR12 galaxy sample, Mon. Not. Roy. Astron. Soc. 470 (2017) 2617 [1607.03155].

[91] SDSS collaboration, M. Betoule et al., Improved cosmological constraints from a joint analysis of the SDSS-II and SNLS supernova samples, Astron. Astrophys. 568 (2014) A22 [1401.4064].

[92] D. Blas, J. Lesgourgues and T. Tram, The Cosmic Linear Anisotropy Solving System (CLASS) II: Approximation schemes, JCAP 1107 (2011) 034 [1104.2933].

[93] B. Audren, J. Lesgourgues, K. Benabed and S. Prunet, Conservative Constraints on Early Cosmology: an illustration of the Monte Python cosmological parameter inference code, JCAP 1302 (2013) 001 [1210.7183]. 
[94] T. Brinckmann and J. Lesgourgues, MontePython 3: boosted MCMC sampler and other features, 1804.07261.

[95] A. Lewis, GetDist: a Python package for analysing Monte Carlo samples, 1910.13970.

[96] Virgo Consortium collaboration, R. E. Smith, J. A. Peacock, A. Jenkins, S. D. M. White, C. S. Frenk, F. R. Pearce et al., Stable clustering, the halo model and nonlinear cosmological power spectra, Mon. Not. Roy. Astron. Soc. 341 (2003) 1311 [astro-ph/0207664].

[97] R. Takahashi, M. Sato, T. Nishimichi, A. Taruya and M. Oguri, Revising the Halofit Model for the Nonlinear Matter Power Spectrum, Astrophys. J. 761 (2012) 152 [1208.2701].

[98] V. Poulin, T. L. Smith, D. Grin, T. Karwal and M. Kamionkowski, Cosmological implications of ultralight axionlike fields, Phys. Rev. D98 (2018) 083525 [1806.10608].

[99] G. Ballesteros and J. Lesgourgues, Dark energy with non-adiabatic sound speed: initial conditions and detectability, JCAP 1010 (2010) 014 [1004.5509].

[100] N. Arendse et al., Cosmic dissonance: new physics or systematics behind a short sound horizon?, 1909.07986.

[101] M.-Z. Lyu, B. S. Haridasu, M. Viel and J.-Q. Xia, $H_{0}$ Reconstruction with Type Ia Supernovae, Baryon Acoustic Oscillation and Gravitational Lensing Time-Delay, 2001.08713.

[102] J. Sakstein and M. Trodden, Early dark energy from massive neutrinos - a natural resolution of the Hubble tension, Phys. Rev. Lett. 124 (2020) 161301 [1911.11760].

[103] F. Niedermann and M. S. Sloth, New Early Dark Energy, 1910.10739.

[104] B. Ratra and P. Peebles, Cosmological Consequences of a Rolling Homogeneous Scalar Field, Phys. Rev. D 37 (1988) 3406.

[105] A. R. Liddle and R. J. Scherrer, A Classification of scalar field potentials with cosmological scaling solutions, Phys. Rev. D 59 (1999) 023509 [astro-ph/9809272]. 